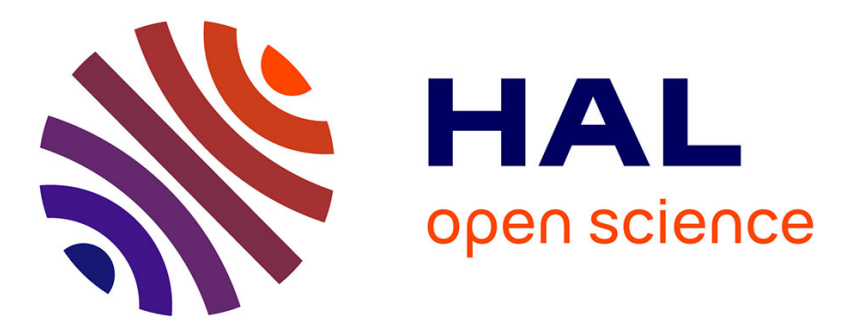

\title{
Irreversible Deformation Mechanisms for 1,3,5-Triamino-2,4,6-Trinitrobenzene Single Crystal through Molecular Dynamics Simulations
}

\author{
Paul Lafourcade, Christophe Denoual, Jean-Bernard Maillet
}

\section{To cite this version:}

Paul Lafourcade, Christophe Denoual, Jean-Bernard Maillet. Irreversible Deformation Mechanisms for 1,3,5-Triamino-2,4,6-Trinitrobenzene Single Crystal through Molecular Dynamics Simulations. Journal of Physical Chemistry C, 2018, 122 (26), pp.14954-14964. 10.1021/acs.jpcc.8b02983 . hal03417483

\author{
HAL Id: hal-03417483 \\ https://hal.science/hal-03417483
}

Submitted on 29 Nov 2021

HAL is a multi-disciplinary open access archive for the deposit and dissemination of scientific research documents, whether they are published or not. The documents may come from teaching and research institutions in France or abroad, or from public or private research centers.
L'archive ouverte pluridisciplinaire HAL, est destinée au dépôt et à la diffusion de documents scientifiques de niveau recherche, publiés ou non, émanant des établissements d'enseignement et de recherche français ou étrangers, des laboratoires publics ou privés.

\section{(ㅇ)(1) $\$$}

Distributed under a Creative Commons Attribution - NonCommerciall 4.0 International 


\section{Irreversible Deformation Mechanisms for}

\section{1,3,5-Triamino-2,4,6-Trinitrobenzene Single}

\section{Crystal Through Molecular Dynamics}

\section{Simulations}

Paul Lafourcade, ${ }^{\dagger, \ddagger}$ Christophe Denoual, ${ }^{*, \dagger}$ and Jean-Bernard Maillet ${ }^{\dagger}$

CEA-DAM, DIF, F-91297 Arpajon, France, and PIMM, Arts et Métiers ParisTech, 151, boulevard de l'Hôpital, F-75013 Paris, France

E-mail: christophe.denoual@cea.fr

${ }^{*}$ To whom correspondence should be addressed

${ }^{\dagger}$ CEA-DAM, DIF, F-91297 Arpajon, France

${ }^{\ddagger}$ PIMM, Arts et Métiers ParisTech, 151, boulevard de l’Hôpital, F-75013 Paris, France 


\begin{abstract}
The plastic behavior of the insensitive energetic molecular crystal 1,3,5-triamino2,4,6-trinitrobenzene (TATB) is investigated through molecular dynamics simulations. A recent method, built to follow any prescribed deformation path, is used to apply directional shear and compressive deformations to a TATB single crystal, leading to the tridimensional characterization of its nucleation von Mises stresses $\sigma_{\mathrm{v}}(\theta, \phi)$, where $\theta$ and $\phi$ are the two angles (latitude and longitude, respectively) that define the loading direction. Furthermore, the local computation of the deformation gradient tensor helps to identify the mechanisms of the irreversible deformation. Two main types of plasticity mechanisms have been identified for the TATB single crystal: first, molecular dynamics simulations predict the existence of dislocations with an unusual local through-plane dilatancy process. Various slip systems among four different non-basal planes have been identified, namely $(\overline{\mathbf{1}} \mathbf{0 1}),(\mathbf{1 0 1}),(\mathbf{0} \overline{\mathbf{1}} \mathbf{1})$ and $(\mathbf{0 1 1})$ planes. Secondly, every deformation containing a basal-plane compressive component involves buckling deformation. A deformation path allowing a perfect twinning of the TATB triclinic cell has been found. This structure has been verified through molecular dynamics (MD) simulations. In order to understand the buckling mechanism, the TATB single crystal behavior under compression along its basal plane is studied in detail.
\end{abstract}

\title{
1 INTRODUCTION
}

1,3,5-triamino-2,4,6-trinitrobenzene (TATB), a well-known highly insensitive energetic molecular crystal, is used in various explosive formulations. It crystallizes in a triclinic cell of space group $\mathrm{P} \overline{1}^{1,2}$ and exhibits very anisotropic thermo-mechanical and chemical behavior ${ }^{3,4}$ due to the arrangement in layers of the two $\mathrm{C}_{6} \mathrm{H}_{6} \mathrm{~N}_{6} \mathrm{O}_{6}$ molecules (see Figure 1). Such anisotropic properties are also the consequences of the directional interactions within the molecular crystal, which give to the strength a high sensitivity toward the direction of loading (up to a factor 20 for shear), as indicated by computations of the second order stiffness tensor $\boldsymbol{C}^{{ }^{5-7}}$ 


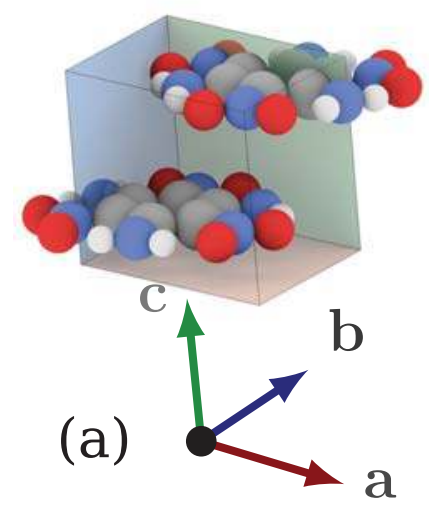

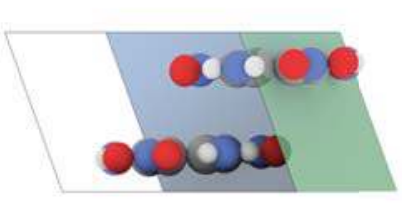

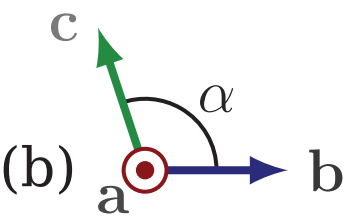

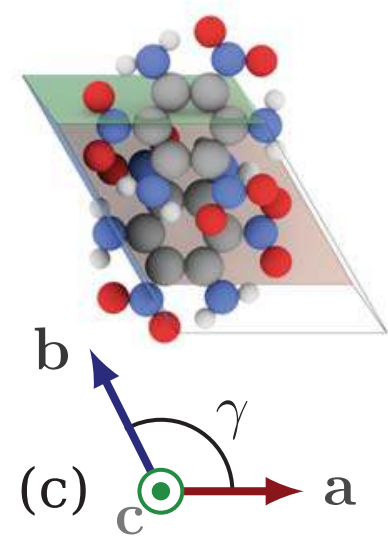

(d)
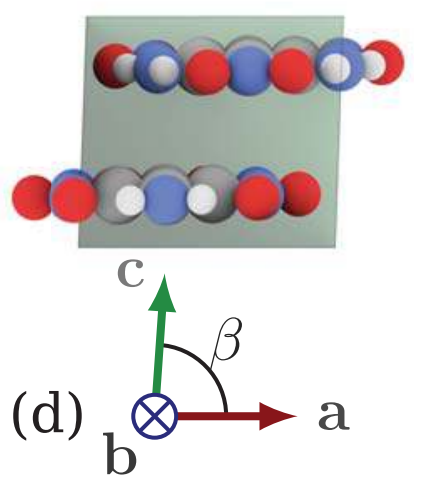

Figure 1: Crystal structure. TATB unit cell with lattice vectors $\mathbf{a}, \mathbf{b}, \mathbf{c}$ and cell angles $\alpha$, $\beta$ and $\gamma$. (a) $3 D$ representation. (b) $x+$ view. (c) $z+$ view. (d) $y+$ view.

Experiments have pointed out that TATB grown single crystals are noticeably defective ${ }^{8-12}$ with the presence of porosities, twinned structures, etc. Until recently TATB single crystals of size compatible with standardized mechanical experiments (e.g. tensile loading) could not be obtained, mainly due to the complexity of the synthesis process. However, Taw et al. ${ }^{13}$ managed to obtain quasihexagonal plate-like crystals of up to $1 \mathrm{~mm}$ width with a minimal presence of impurities. They were able to perform nanoindentation experiments on as-grown TATB single crystals with sizes varying between $200 \mu \mathrm{m}$ and $600 \mu \mathrm{m}$ which represents a major step in the experimental area for this material, although the process used differs from the typical manufacturing one.

Molecular Dynamics (MD) simulations of TATB single crystal is widely used and plays a crucial part in predicting its macroscopic behavior. Kroonblawd et al..$^{3,4,14}$ focused their work on the thermal behavior of TATB and relaxation of idealized hot spots. Mathew et al. computed the first generalized stacking fault surfaces of TATB ${ }^{6}$ and presented MD simulations of its elastic-plastic response under displacement-controlled nanoindentation. ${ }^{15}$ The latter results are shown to be in very good agreement with the recent experimental work of Taw et al. ${ }^{13}$ Since plasticity in crystalline materials is known to be mainly controlled by dislocations, Lafourcade et al. ${ }^{7}$ studied the structure of such defects in TATB basal plane. 
They computed the dislocation core structures as a function of pressure and temperature and demonstrated that dislocations split into three or more partials that spread over approximatively 50 to 100 unit cells. Thus, dislocations in TATB basal plane must be described as large stacking fault ribbons rather than discrete dislocation lines.

The ability of High Energetic (HE) materials to remain stable during shock loading defines their sensitivity, a critical property for the safety of such materials. This sensitivity is highly dependent on complex mechanisms such as localizations of deformation ${ }^{16}$ which are one aspect of hot-spots. ${ }^{15,17-19}$ Such hot spots are known to be triggered by local plastic deformations, dislocation pile-up, ${ }^{15}$ void collapse ${ }^{20,21}$ or localized heating due to defects nucleation. ${ }^{17}$ Irreversible deformation mechanisms thus need to be accurately described for a predictive modeling of ignition.

In this paper, we present a simulation set-up dedicated to the analysis of the onset and propagation of irreversible deformation mechanisms in TATB, for prescribed deformation paths. Stress-strain curves are obtained for pure shear and uniaxial loadings, with direction spanning all possible orientations. A detailed analysis of mechanisms of irreversible evolution is proposed by a local estimate of the deformation gradient tensor, a Lagrangian objective measure of deformation.

The paper is organized as follows. In the first section, we present the two main methods used in our MD simulations for this work: the first depicts a way to prescribe the deformation paths while the second describes the implementation of the local measure of the deformation gradient tensor. The second section is dedicated to the investigation of the plasticity mechanisms and their dependence on the direction of loading. The two main mechanisms are then presented in detail: the dislocation nucleation on non-basal slip systems for which an inventory is proposed, and the onset of a microstructure analogous to twin bands in metals that results from in-plane compression. 


\section{METHODS}

The STAMP $\operatorname{code}^{22}$ was used to perform all the MD simulations with the non-polarizable, $20 \%$ scaled-charges all-atoms force field for TATB developed by Bedrov et al. ${ }^{5}$ with planar, symmetric rigid molecules. Long-range electrostatic interactions were computed using the Reaction Field ${ }^{23-25}$ approximation with a 13 A cutoff and 100.0 for the dielectric constant $\varepsilon$ instead of the Ewald summation. All other interactions were computed with a cut-off distance of $13 \AA$. A Langevin thermostat was used with the damping constant set to $1.0 \mathrm{ps}$. The time step for integrating the equations of motion was set to 1.0 fs for both isochoric-isoenergetic (NVE) and isochoric-isothermal (NVT) simulations, a time step consistent with the choice of using rigid molecules. Finally, the rigid body dynamics is computed by discretization of the equations of motion following a Velocity-Verlet scheme. ${ }^{26}$ This force field has been used in many studies to compute the dependence on temperature and pressure of TATB elastic constants, ${ }^{5-7}$ thermal conductivity, ${ }^{3,4} \gamma$-surfaces,,${ }^{6,7}$ nanoindentation, ${ }^{15}$ hot spots, ${ }^{27}$ within DPDE shock loading on (001) planes $^{14}$ and more recently dislocation core structures. ${ }^{7}$

\subsection{Deformation Paths}

Due to the high anisotropy of TATB, the mechanisms of irreversible deformation strongly depend on the direction of loading. The understanding of plastic activity and mechanical behavior of TATB single crystal is important at the atomistic scale if one wants to build a constitutive law at larger time and space scales. To be able to detect all possible mechanisms, we propose a methodology to perform large deformation 3D-periodic MD simulations. ${ }^{28}$ In order to apply any type of deformation (pure shear, pure compression, compression with lateral distension, etc.) along any direction of the 3D space, we prescribe a velocity gradient $\boldsymbol{L}=\dot{\boldsymbol{F}} \cdot \boldsymbol{F}^{-1}$ defined by a deformation gradient tensor $\boldsymbol{F}(t)$ and its time derivative $\dot{\boldsymbol{F}}$. The deformation gradient tensor $\boldsymbol{F}$ is constructed as follows: 


$$
\boldsymbol{F}=\boldsymbol{I}+\alpha(t) \boldsymbol{m} \otimes \boldsymbol{m}+\beta(t) \boldsymbol{n} \otimes \boldsymbol{n}
$$

where $\alpha$ and $\beta$ are time dependent functions, $\otimes$ is the dyadic product $\left(\boldsymbol{a} \otimes \boldsymbol{b}=a_{i} b_{j}\right),(\boldsymbol{m}, \boldsymbol{n})$ are the vectors used for the construction of $\boldsymbol{F}$ as described in Figure 2 and $\boldsymbol{I}$ is the identity tensor. Two types of loadings are used in the following: pure shear strain is obtained by taking $\beta=-\alpha /(1+\alpha)$ (implying that $\operatorname{det} \boldsymbol{F}=1$ ), and uniaxial loading compression with $\beta=0$.

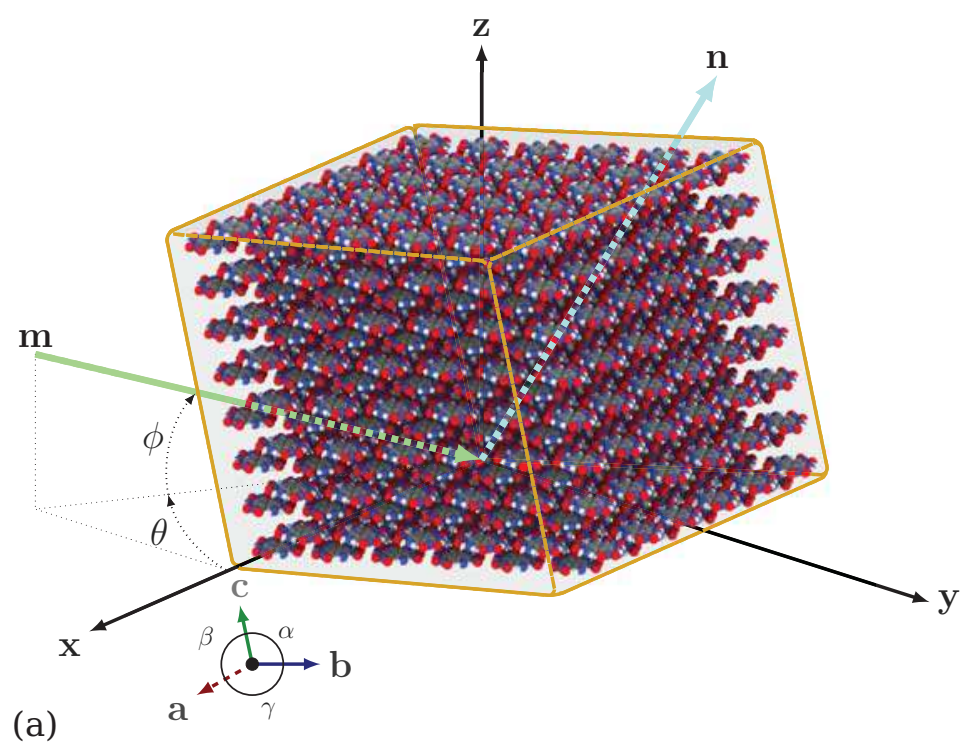

$$
\begin{gathered}
\mathbf{m}=\left[\begin{array}{c}
\cos \theta \cos \phi \\
\sin \theta \cos \phi \\
\sin \phi
\end{array}\right] \\
\mathbf{n}=\left[\begin{array}{c}
\cos \theta \sin \phi \\
\sin \theta \sin \phi \\
\cos \phi
\end{array}\right]
\end{gathered}
$$

(b)

Figure 2: (a) Space configuration of the large strain context used in this work. $(\boldsymbol{x}, \boldsymbol{y}, \boldsymbol{z})$ is the reference orthonormal system. $(\boldsymbol{m}, \boldsymbol{n})$ leads to the construction of the gradient deformation tensor $\boldsymbol{F}$. $(\boldsymbol{a}, \boldsymbol{b}, \boldsymbol{c})$ is the crystal unit cell system. (b) Definition of $\boldsymbol{m}$ and $\boldsymbol{n}$ in the reference orthonormal system with respect to angles $\theta$ and $\phi$.

For each MD simulations discussed in this paper, a 100 ps trajectory was calculated in the NVT ensemble in order to reach equilibrium, using cell parameters obtained from a Parrinello-Rahman isostress-isothermal ( $\mathrm{N} \sigma \mathrm{T})$ simulation (at the same temperature). The final configuration is then used as initial conditions for subsequent MD simulations with deformations (i.e. $t=0$ ). Then, the MD supercell is dynamically strained, knowing its average deformation through the application of $\boldsymbol{F}$ to the $(\boldsymbol{a}, \boldsymbol{b}, \boldsymbol{c})$ system's boundaries only (see Figure 2). A velocity field is applied at initial time (i.e. after $100 \mathrm{ps}$ of equilibration) and is consistent with the velocity gradient tensor $\boldsymbol{L}$ deduced from $\boldsymbol{F}($ i.e., $\vec{v}(t=0)=\boldsymbol{L} \cdot \vec{x}(t=0))$. 
No rescaling of the atomic positions are used in this method, which allows for a consistent motion of molecules regarding the applied strain and avoids the generation of acoustic waves in the material.

The aformentioned deformation, applied by dynamic modification of cell parameters, may lead to an irreversible evolution of the system through the appearance of local defects. Two different analyses were used to capture these processes.

When full dislocations (nucleation and motion) form, the original crystal structure is recovered behind the moving dislocation but is strongly perturbed at the dislocation core. Local detection of loss of crystalline structure (as the one proposed, e.g., in the DXA algorithm $^{29}$ ) highlights the dislocation line, but not the dislocation slip plane. For defects such as twins, the local perturbation of crystal order only gives information on the location of twins' mirror plane. Such analysis focuses on the instantaneous state of the crystal (dislocations lines, twins boundaries, etc), without indicating the history of the deformation.

Frobenius norm of local Green-Lagrange tensor, on the other hand, provides a measure of the distance (in terms of deformation) between the initial state (no defect) and the actual microstructure. A dislocation loop is highlighted through the formation of a pennyshaped disregistry, representing the deformation induced by the dislocation motion. Local Lagrangian deformation gradient tensor $\hat{\boldsymbol{F}}$, when described as a composition of rotation $\hat{\boldsymbol{R}}$ and stretch $\hat{\boldsymbol{U}}$ tensors (polar decomposition $\hat{\boldsymbol{F}}=\hat{\boldsymbol{R}} \cdot \hat{\boldsymbol{U}}$ ) provides a means to distinguish zones that have undergone a reconstructive (i.e. irreversible and dissipative) deformation from zones that have only rotated (i.e. reversible deformation with no dissipation).

For these reasons, the computation of the local Lagrangian deformation gradient tensor $\hat{\boldsymbol{F}}$ with respect to a reference defect-free state is preferred in the following. This measure has been proposed by several authors (Gullett et al., ${ }^{30}$ Tucker et al., ${ }^{31}$ Denoual et al. ${ }^{32}$ ) and more recently by Kober et al. ${ }^{33}$ based on strain functionals. The computational details can be found in the appendix. 


\section{RESULTS}

\subsection{Nucleation Stress Surface Under Pure Shear}

\subsubsection{Simulation Setup}

MD simulations were performed with a $3 D$-periodic $10 \boldsymbol{a} \times 10 \boldsymbol{b} \times 14 \boldsymbol{c}$ simulation cell containing 2800 molecules. Cell parameters at different temperatures and pressures were previously obtained through Parrinello-Rahman simulations. At first, the system is equilibrated in the NVT ensemble. Then, the large strain methodology presented in 2.1 to apply directional pure shear to the simulation box is used. The plane defined by the two vectors $\boldsymbol{m}$ and $\boldsymbol{n}$ is chosen to be perpendicular to the basal plane of TATB. Two conditions are applied concurrently for these simulations: volume-preserving strains are applied at constant strain rate, in order to construct the following expression for the deformation gradient tensor, depending on time $t$ :

$$
\boldsymbol{F}=\boldsymbol{I}+\left(e^{A t}-1\right) \boldsymbol{m} \otimes \boldsymbol{m}+\left(e^{-A t}-1\right) \boldsymbol{n} \otimes \boldsymbol{n}
$$

with $A=\ln (C) / t_{\text {tot }}$, where $C$ is the final deformation ratio and $t_{\text {tot }}$ the total simulation

time. Vectors $\boldsymbol{m}$ and $\boldsymbol{n}$ represent the compression and expansion in orthogonal directions. In Equation 2, the coefficients ahead of each dyadic product ensure that this deformation corresponds exactly to a pure shear (i.e. the amount of compression along $\boldsymbol{m}$ equals the amount of expansion along $\boldsymbol{n})$.

\subsubsection{Results at $(300 \mathrm{~K}, 0 \mathrm{GPa})$}

The mechanisms of irreversible deformation were identified and characterized at $300 \mathrm{~K}$ and $0 \mathrm{GPa}$. Values of $\theta$ and $\phi$ were picked up every $30^{\circ}$ in $[0,2 \pi]$ and every $15^{\circ}$ in $[0, \pi / 2]$, respectively. By doing this, 84 different deformation paths (corresponding to 84 distinct pairs of $\theta$ and $\phi$ ) were examined through MD simulations, at a strain rate equal to $10^{8} \mathrm{~s}^{-1}$. The final deformation ratio was set to 0.9 for each deformation path, leading to a final 


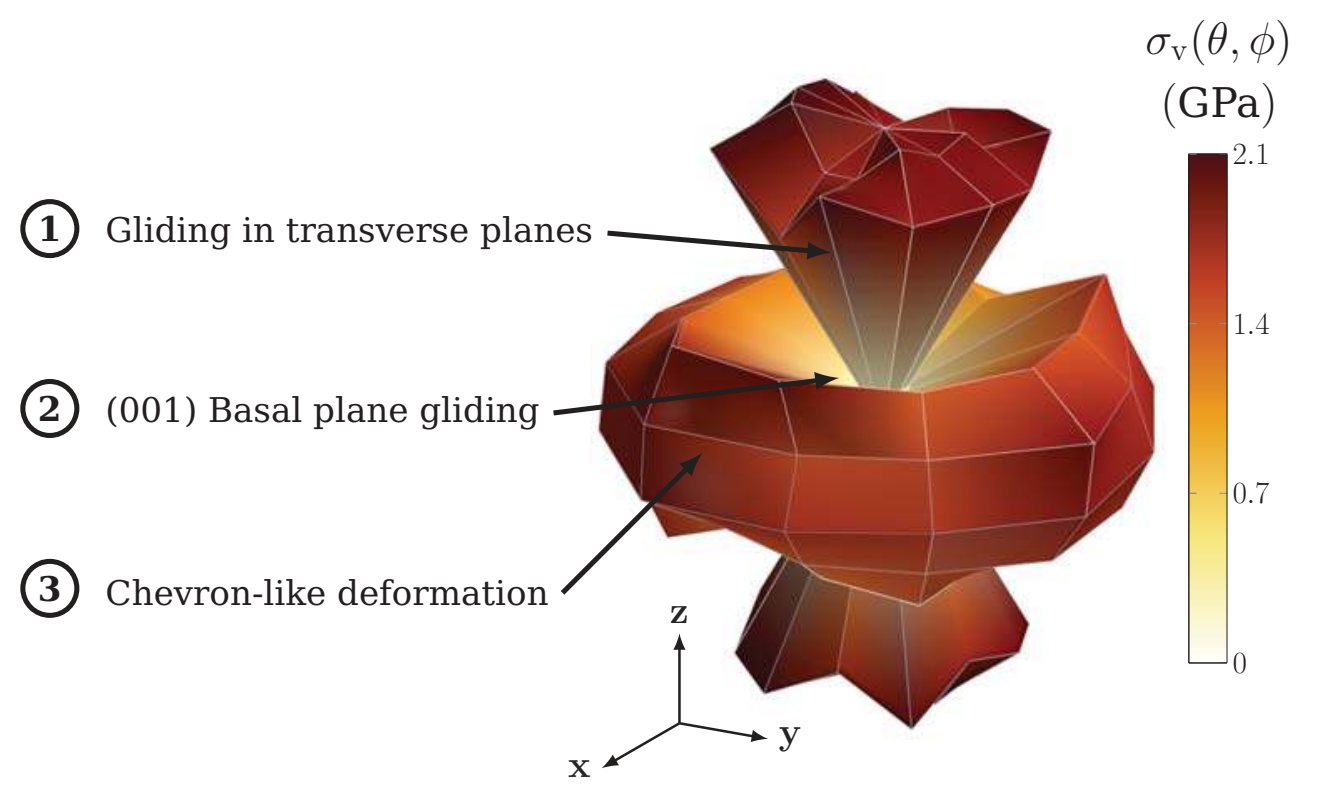

Figure 3: 3D representation of TATB nucleation von Mises stress $\sigma_{\mathrm{v}}(\theta, \phi)$ at nucleation under pure shear loading with identification of the three main mechanisms that occur. Color coding is based on the value of $\sigma_{\mathrm{v}}(\theta, \phi)$.

equivalent deformation of $10 \%$.

For each trajectory, a drop in the evolution of the stress with time is observed, which we interpreted as signalling the onset of an irreversible deformation. Maximum von Mises stress before the drop defines the time to nucleation and the stress to nucleation $\sigma_{\mathrm{v}}(\theta, \phi)$.

Taking into account the TATB single crystal centrosymmetry, a 3D surface representation of $\sigma_{\mathrm{v}}(\theta, \phi)$ is shown in Figure 3. One can identify three distinct zones on this surface, numbered 1 to 3 .

In the zone labelled 1 in Figure 3, which corresponds to $\phi>45^{\circ}$, the gliding of the basal plane is not (or weakly) activated and most of the deformation is accommodated by nucleation of dislocations with non-basal Burgers vectors. The onset of these dislocations induces a hydrogen-bond breaking phenomenon with an unusual local dilatancy (see Figure 4a), investigated and explained in detail in Section 3.2.

The zone related to the lowest value of $\sigma_{\mathrm{v}}$ (label 2) corresponds to the deformation paths where $\phi=45^{\circ}$ (number 2 in Figure 3). The deformation is equivalent to a pure shear (see Equation 2) in the basal plane (001). Glide is activated for stress below computable accuracy, 

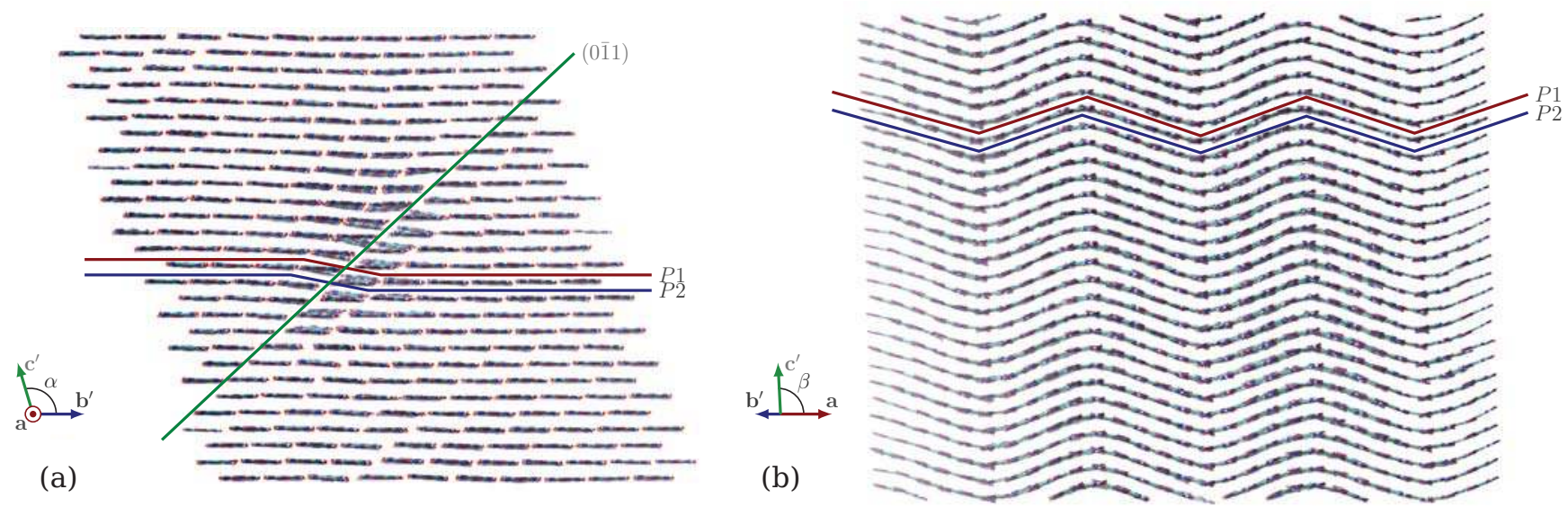

Figure 4: All-atom display with identification of basal $(P 1, P 2)$ and transverse planes $(0 \overline{1} 1)$ of interest. (a) TATB single crystal non-basal gliding in the (011) plane for a deformation that consists in a pure shear along this plane. (b) Buckling mechanism of TATB single crystal for a deformation that mainly contains a compressive component along the [100] direction. The prime $\left(^{\prime}\right)$ symbol on the vector labels means that these vectors do not lie in the plane of the figure.

owing to the weak van der Waals interactions between layers, which leads to the low energy barriers observed in the $\gamma$-surfaces. ${ }^{6,7}$

Finally, in the zone 3 in Figure 3 that looks like a "donut", the deformation is mainly compressive in the basal plane, inducing an alternating disorientation of molecular layers relative to their initial planar configuration (see Figure 4b). This mechanism has already been observed in oriented block copolymers. ${ }^{34}$ An analysis of this phenomenon is provided in Section 3.3.

It is important to note that non-basal plane dislocations and buckling deformation mechanism are triggered for similar values of the nucleation von Mises stress at standard ambient conditions.

\subsubsection{Evolution With Pressure}

In the following, we study the evolution of the nucleation stress with pressure. The cell parameters of TATB single crystal were obtained at $300 \mathrm{~K}$ and for different pressures $(2$, 5, 10 and $20 \mathrm{GPa}$ ) using Parrinello-Rahman simulations. To reduce the computation cost we select trajectories with $\theta=90^{\circ}$ and values of $\phi$ picked up every $15^{\circ}$ in $[0, \pi / 2]$. Since 
at ambient conditions the TATB behavior is weakly dependent on $\theta$, these measures give a good insight of the behavior under pressure.

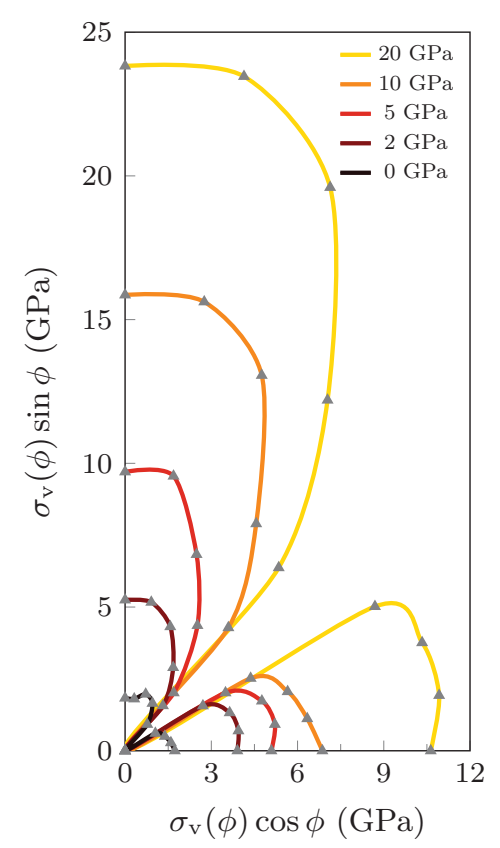

Figure 5: Evolution with pressure of the nucleation von Mises stress $\sigma_{\mathrm{v}}(\phi)\left(\theta=90^{\circ}\right)$. Gray triangles correspond to the simulation data, curves are interpolation to guide the eye.

The setup for the deformation paths in pressure was the same as the one used at standard ambient conditions. The results are presented in Figure 5. The angle $\phi=45^{\circ}$ (a pure shear in the basal plane, corresponding to zone 2 in Figure 3) has a very low threshold stress (below computable accuracy) for all pressures. The significant increase of the nucleation stress for the onset of dislocations (zone 1 in Figure 3) with respect to the pressure is consistent with the dilatancy of the dislocation core that could hinder the nucleation process as the pressure increases.

\subsubsection{Size and Strain Rate Effects}

In the simulation described above, system size effects may have impacted the nucleation stress threshold. This must be understood in order to be able to build a constitutive law at higher time and space scales. The applied strain rate was very high compared to what one would expect during experiments on an engineering scale but more appropriate compared 
to the local material strain rate in a shock rise. We have chosen to carry out additional trajectories at a lower strain rate in order to investigate the effect of strain rate on the threshold of the two main mechanisms described above.

First, we have performed three trajectories while increasing the system size. Taking the cell parameters at $300 \mathrm{~K}$ and $0 \mathrm{GPa}, \mathrm{MD}$ simulations were performed with a $3 D$-periodic $40 \boldsymbol{a} \times 40 \boldsymbol{b} \times 60 \boldsymbol{c}$ simulation cell containing 179200 molecules. Deformation paths were performed with $\theta=90^{\circ}$ and for three different values of $\phi: 15^{\circ}, 75^{\circ}$ and $90^{\circ}$. The strain rate was the same as for the simulations at (300 K, 0 GPa).

Table 1: Size effect on the nucleation stress $\sigma_{\mathrm{v}}(\theta, \phi)$ for fixed $\theta=90^{\circ}$

\begin{tabular}{cccc}
\hline & $10 \boldsymbol{a} \times 10 \boldsymbol{b} \times 14 \boldsymbol{c}$ & $40 \boldsymbol{a} \times 40 \boldsymbol{b} \times 56 \boldsymbol{c}$ & ratio \\
$\phi\left(^{\circ}\right)$ & $\sigma_{\mathrm{v}}(\mathrm{GPa})$ & $\sigma_{\mathrm{v}}(\mathrm{GPa})$ & $(\%)$ \\
\hline 15 & 1.618 & 1.346 & $\simeq 20.2$ \\
75 & 2.448 & 2.097 & $\simeq 16.7$ \\
90 & 2.269 & 1.834 & $\simeq 23.7$
\end{tabular}

The aim of these three simulations was to quantify the size effects on the nucleation stresses. The different values of the von Mises stress at the nucleation of the first defect are reported in Table 1. As anticipated, increasing the size of the system decreases the nucleation stress threshold. By multiplying the system size by 4 in each direction, the von Mises stress at nucleation has been lowered by approximately 20\%, regardless of the loading direction.

In order to study the effect of the strain rate, additional trajectories were undertaken with the same system size as before (i.e. $10 \boldsymbol{a} \times 10 \boldsymbol{b} \times 14 \boldsymbol{c})$. Deformation paths were chosen with $\theta=0^{\circ}$ and $\phi$ every $15^{\circ}$ in $[0, \pi / 2]$, as for the computation of the nucleation stresses surface in Figure 3. The results are shown in Table 2, where one can see that the lower strain rate reduces the nucleation stress threshold by $14 \%$. 
Table 2: Strain rate effect on the nucleation stress $\sigma_{\mathrm{v}}(\theta, \phi)$ for fixed $\theta=0^{\circ}$

\begin{tabular}{cccc}
\hline$\phi\left({ }^{\circ}\right)$ & $\begin{array}{c}10^{8} \mathrm{~s}^{-1} \\
\sigma_{\mathrm{v}}(\mathrm{GPa})\end{array}$ & $\begin{array}{c}10^{7} \mathrm{~s}^{-1} \\
\sigma_{\mathrm{v}}(\mathrm{GPa})\end{array}$ & $\begin{array}{c}\text { ratio } \\
(\%)\end{array}$ \\
\hline 0 & 1.612 & 1.407 & $\simeq 12.7$ \\
15 & 1.409 & 1.215 & $\simeq 13.8$ \\
30 & 1.249 & 1.082 & $\simeq 13.4$ \\
60 & 1.699 & 1.458 & $\simeq 14.2$ \\
75 & 1.781 & 1.537 & $\simeq 13.7$ \\
90 & 1.833 & 1.593 & $\simeq 13.1$
\end{tabular}

\subsection{Non-basal Dislocations}

In this section, we propose a description of possible slip systems in the identified planes during MD simulations, and we explain the local dilatancy process that comes with the transverse gliding.
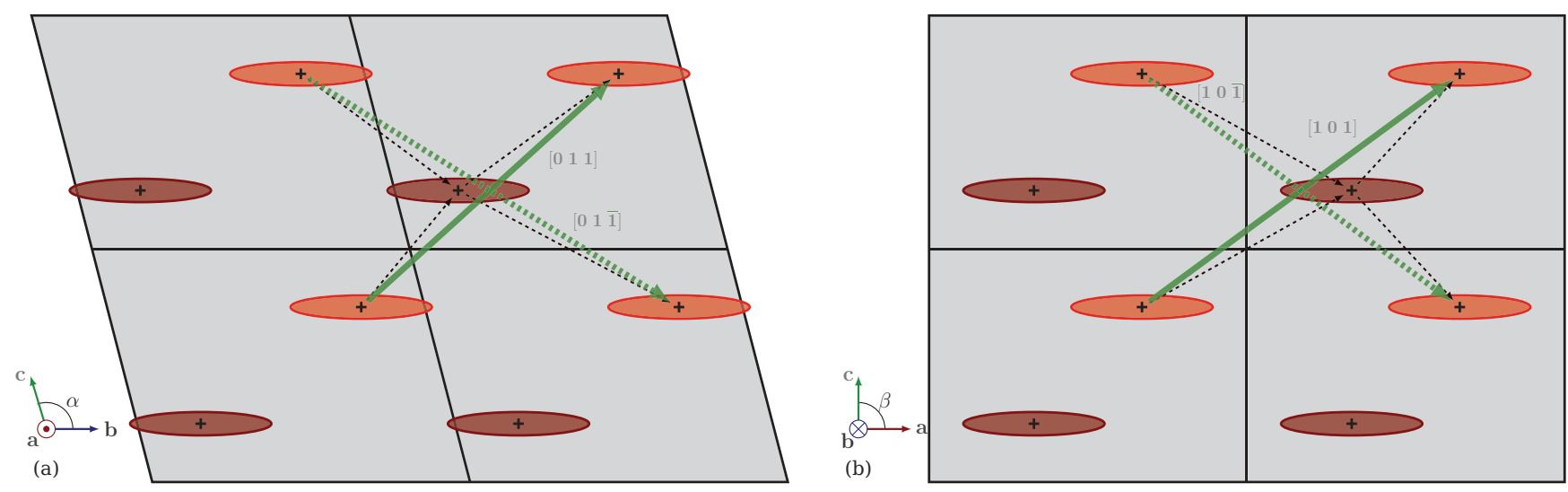

Figure 6: Review of potential unit slip vectors in transverse planes of TATB single crystal. (a) View along [100] direction. (b) View along [010] direction. Brown and orange shapes represent TATB molecules. Orange molecules are in the figure plane whereas brown ones are located behind it. Burgers vectors are represented as green continuous arrows and partial slip vectors as black dashed arrows.

\subsubsection{Potential Slip Systems}

Based on the crystal structure and positions of molecular centers of mass, one can identify the different potential unit slip vectors for dislocations in TATB single crystal. Figure 6 contains 
views along the normal of the two non-basal planes, namely (100) and (010), defined by $\boldsymbol{b} \times \boldsymbol{c}$ and $\boldsymbol{c} \times \boldsymbol{a}$, respectively. Green continuous arrows represent the Burgers vectors whereas black dashed arrows identify partial slip vectors that interchange the planes $P 1$ and $P 2$.

The two slip systems in the (001) planes have already been studied, first by Mathew and Sewell ${ }^{6}$ who computed the related $\gamma$-surfaces and then by Lafourcade et al. ${ }^{7}$ who reported the first estimate of the dislocation core structure. Lafourcade et al. concluded that due to the easy gliding in basal planes, dislocations can not be considered as discrete lines but rather as large stacking fault ribbons.

The planes (100) and (010) are of similar nature and can be studied together. Among all possible Burgers vectors for dislocation nucleation within (100) and (010) planes, [100], [010] and [001] Burgers vectors were never observed and we think that this type of gliding can be dismissed, due to the large steric hindrance present within the molecular layers. However, the gliding with a [001] component (thus implying hydrogen-bond breaking) in transverse planes has been observed in our simulations and occurs via formation of a complex stacking fault structure that entangles the $P 1$ and $P 2$ planes. The different Burgers vectors are represented as green arrows in Figure 6. This structure leads to a noticeable dilatancy, as explained in the next section.

\subsubsection{Local Dilatancy Process}

In undeformed TATB crystal, the packing of molecules is hexagonal and is ruled by the facing arrangement of $\mathrm{NO}_{2}$ and $\mathrm{NH}_{2}$ groups through strong in-plane hydrogen bonding. Combined with the hexagonal molecular structure, these $\mathrm{NO}_{2}$ and $\mathrm{NH}_{2}$ stabilize the $\gamma$ angle to $120^{\circ}$ in the basal plane as shown by the yellow shape in Figure $7 \mathrm{~b}$.

Taking the system $[0 \overline{11}](0 \overline{1} 1)$ as an example, we note that at half Burgers $\frac{1}{2}[0 \overline{11}]$, the planes $\mathrm{P} 1$ and $\mathrm{P} 2$ line up, so that $\mathrm{NO}_{2}$ are facing $\mathrm{NO}_{2}$ and $\mathrm{NH}_{2}$ are facing $\mathrm{NH}_{2}$ groups, as shown in Figure 7c and 7d. This induces an in-plane dilatancy in every second layer (see Figure $7 \mathrm{a}$, red annotation), that comes with a local strain that transforms the hexagonal 
(a)
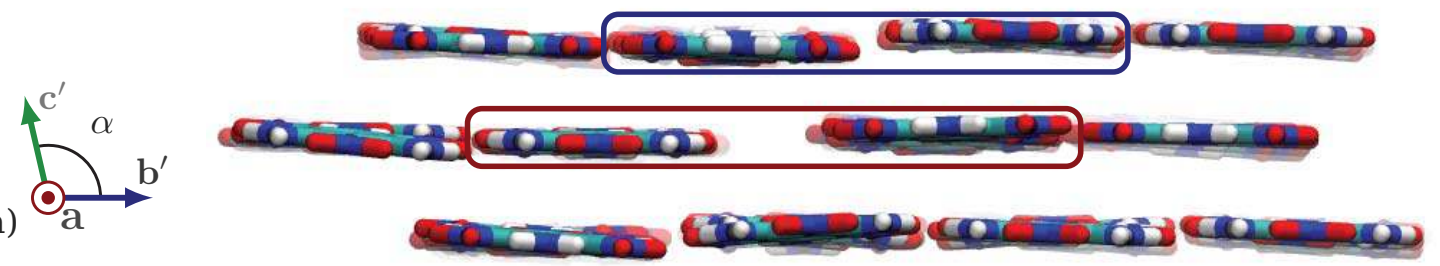

Perfect crystal

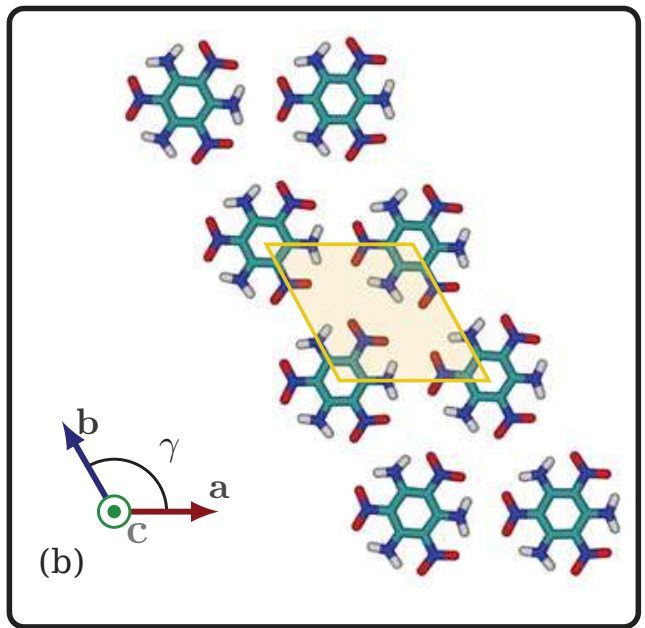

Stacking fault configuration

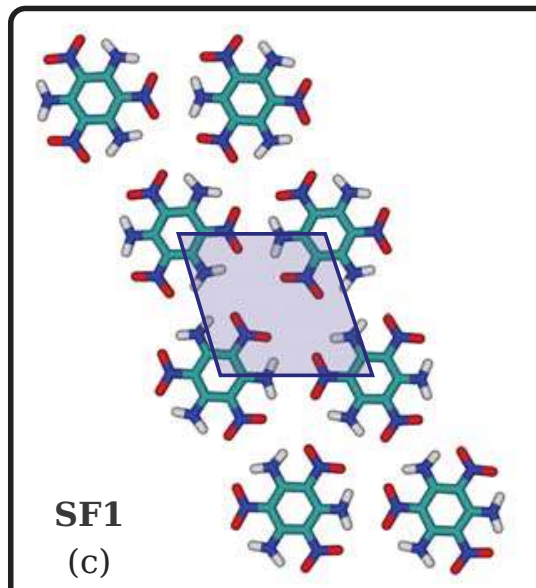

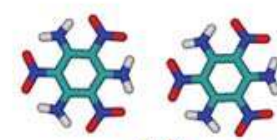

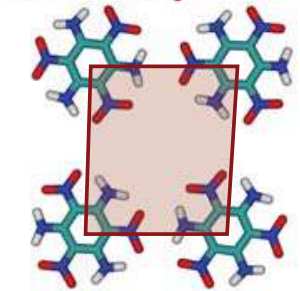

SF2

(d)

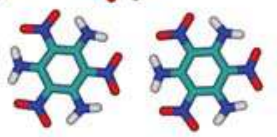

Figure 7: Transverse dislocation process. (a) 100 view of three successive planes during the midway configuration of a $[0 \overline{11}](0 \overline{1} 1)$ dislocation that propagates into a TATB single crystal. Blue and red shapes encircle stacking fault configurations $S F 1$ and $S F 2$ respectively. (b) Molecular arrangement in a defect-free TATB basal plane. (c) and (d) In-plane molecular stacking for the two successive stacking fault configurations in TATB basal plane $S F 1$ and $S F 2$, respectively. Carbon is cyan, nitrogen is blue, oxygen is red and hydrogen is white. 
stacking (yellow annotation in Figure 7b) into a rectangular-like arrangement. For the plane with the lower dilatancy, a strain is also visible, although less stronger (blue annotation in Figure 7c).

\subsubsection{Activated Slip Systems}

The procedure described in Section 3.1 spans all the orientations that can lead to the nucleation of non-basal plane dislocations. Table 3 gives a list of slip system (slip plane + Burgers vector) activated during our MD simulations.

Table 3: Activated Slip Systems in TATB Single Crystal

\begin{tabular}{ccc}
\hline slip system & slip plane normal & slip vector \\
\hline$\left[\begin{array}{lll}\overline{1} & 0 & \overline{1}\end{array}\right]\left(\begin{array}{lll}\overline{1} & 0 & 1\end{array}\right)$ & $\left(\begin{array}{lll}\overline{1} & 0 & 1\end{array}\right)$ & {$\left[\begin{array}{lll}\overline{1} & 0 & \overline{1}\end{array}\right]$} \\
\hline$\left[\begin{array}{lll}1 & 0 & \overline{1}\end{array}\right]\left(\begin{array}{lll}1 & 0 & 1\end{array}\right)$ & $\left(\begin{array}{lll}1 & 0 & 1\end{array}\right)$ & {$\left[\begin{array}{lll}1 & 0 & \overline{1}\end{array}\right]$} \\
\hline$\left[\begin{array}{lll}0 & \overline{1} & \overline{1}\end{array}\right]\left(\begin{array}{lll}0 & \overline{1} & 1\end{array}\right)$ & $\left(\begin{array}{lll}0 & \overline{1} & 1\end{array}\right)$ & {$\left[\begin{array}{lll}0 & \overline{1} & \overline{1}\end{array}\right]$} \\
\hline$\left[\begin{array}{lll}0 & 1 & \overline{1}\end{array}\right]\left(\begin{array}{lll}0 & 1 & 1\end{array}\right)$ & $\left(\begin{array}{lll}0 & 1 & 1\end{array}\right)$ & {$\left[\begin{array}{lll}0 & 1 & \overline{1}\end{array}\right]$}
\end{tabular}

\subsection{Micro-Twinning Deformation}

For deformation paths that contain a strong compressive component in the basal plane, a buckled structure appears as shown in Figure 4b, involving a homogeneous shear of the (001) plane. We first present the geometrical transformation for the twinning in Section 3.3.1. Then, in Section 3.3.2, we propose the computation of an energy landscape that details the energetics during any simple shear in TATB basal plane. Finally, a study of large TATB single crystal plates $(100 \boldsymbol{a} \times 100 \boldsymbol{b} \times 10 \boldsymbol{c}$ MD simulation cells $)$ is undertaken in Section 3.3.3 to characterize the behavior under compression in terms of stability, size, and strain rate dependence. 


\subsubsection{Perfect Twinning}

The triclinic TATB molecular crystal is centrosymmetric with space group $\mathrm{P} \overline{1},{ }^{1}$ and consists of alternating stacking (ABABAB) of molecular layers. The hexagonal-like basal plane symmetry and stacking of the layers leads to a perfect twinning structure that can be obtained (at constant volume and interplanar distance) by an inversion of the out-of-plane angles $\alpha$ and $\beta: \alpha^{\prime}=180-\alpha=91.47^{\circ}, \beta^{\prime}=180-\beta=76.64^{\circ}$. This transformation changes the orientation of the amino and nitro groups in the same layer and the relative coordinates of molecules in the unit cell. A shift of $\frac{1}{2}[\mathbf{0 0 1}]$ (see Figure 8) restores both the molecules amino and nitro groups orientations and the location of the molecule within the cell.
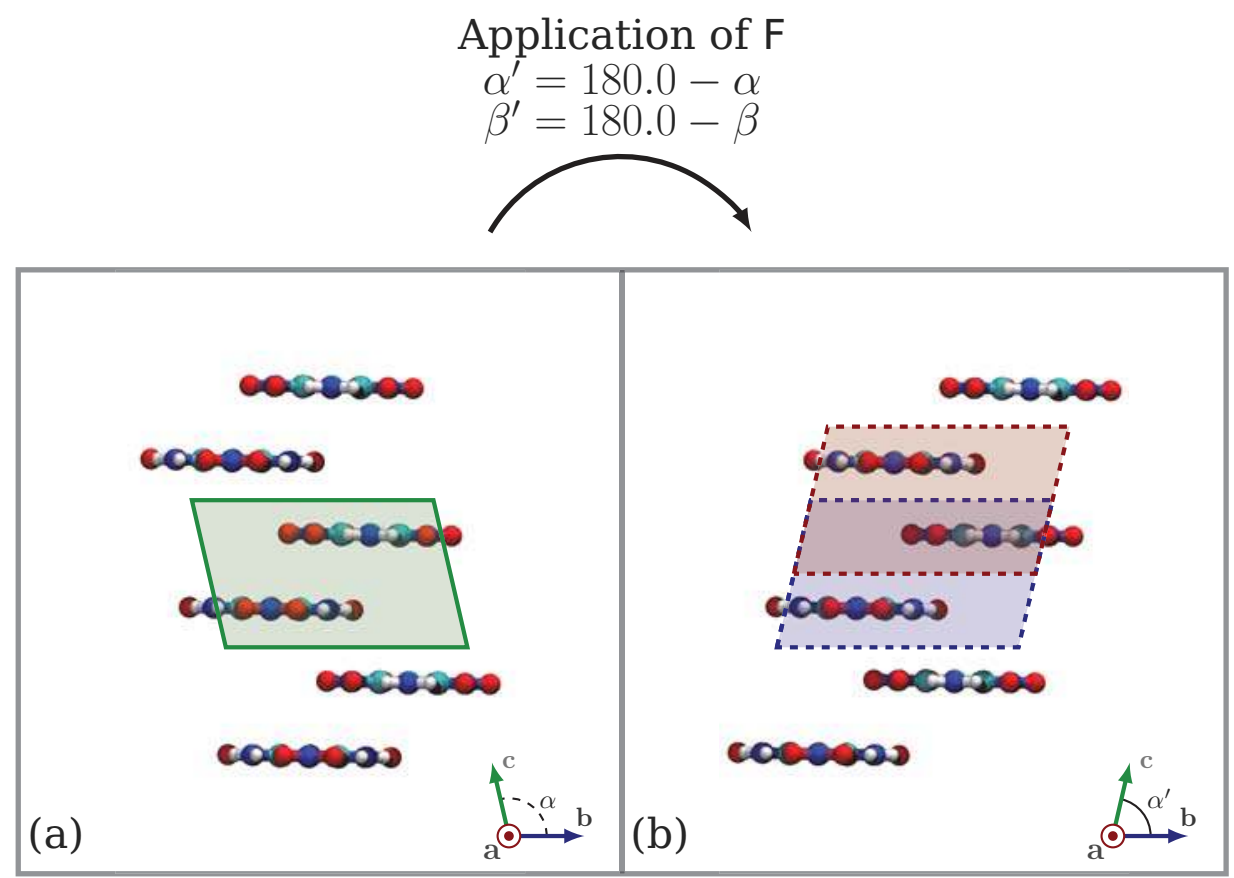

Figure 8: Detailed twinning process for TATB single crystal. (a) Three unstrained TATB triclinic cells. (b) TATB cells after inversion of the two out-of-plane angles $\alpha$ and $\beta$. The unit cell shifted about $\frac{1}{2} \boldsymbol{c}$ (highlighted in red) is the original one after a rotation of $180^{\circ}$ around the $z$-axis. Only the process of the $\alpha$ angle inversion is shown here, which is similar for the $\beta$ angle. Carbon is cyan, nitrogen is blue, oxygen is red and hydrogen is white.

\subsubsection{Homogeneous Shear Deformation}

The energetics along the twinning path is investigated in this section, with a particular focus on possible metastable phases (or twin structures) and the stress needed to nucleate these 
phases. The twinning deformation is a simple shear in the basal plane (001), so that a comprehensive calculation of this energetics can be done by calculating the excess energy after an instantaneous deformation given by:

$$
\boldsymbol{F}=\boldsymbol{I}+\frac{1}{d_{\mathrm{int}}} \boldsymbol{f} \otimes \boldsymbol{n}
$$

where $\boldsymbol{f}$ is the shear vector defined as $\boldsymbol{f}=f_{1} a\left[\begin{array}{lll}\mathbf{1} & \mathbf{0} & \mathbf{0}\end{array}\right]+f_{2} b\left[\begin{array}{lll}\mathbf{0} & \mathbf{1} & \mathbf{0}\end{array}\right]$ with $f_{1}$ and $f_{2}$ varying from -0.5 to 0.5 . $\boldsymbol{n}=\boldsymbol{z}$ is the normal vector to the basal plane, $d_{\text {int }}$ the interplanar distance between molecular layers, assumed constant here, and $\boldsymbol{I}$ is the identity tensor.

This landscape must be calculated at $300 \mathrm{~K}$ to be consistent with the MD simulations done in this study. However, prescribing an overall deformation is not compatible with our scheme to integrate the equation of motion since we want to evaluate the energy for each fraction of the shear vector $\boldsymbol{f}$. Assuming ergodicity for the system, we choose to sample the system over the equilibrium state by considering a large simulation supercell of $20 \boldsymbol{a} \times 20 \boldsymbol{b} \times$ $28 \boldsymbol{c}$ equilibrated in the NVT ensemble during 100 ps (with cell parameterers obtained with the Parrinello-Rahman simulations at $300 \mathrm{~K}$ ). Then, an instantaneous deformation through $\boldsymbol{F}$ is applied in order to evaluate the energetics along the prescribed deformation. A similar procedure has been proposed by Lafourcade et al. ${ }^{7}$ to estimate the TATB basal $\gamma$-surface at $300 \mathrm{~K}$.

The deformation gradient tensor $\boldsymbol{F}$ corresponding to this homogeneous deformation has only two non-trivial components $F_{13}$ and $F_{23}$, functions of $f_{1}$ and $f_{2}$. Considering the right Cauchy-Green tensor $\boldsymbol{C}=\boldsymbol{F}^{\mathrm{T}} \cdot \boldsymbol{F}$ so as to eliminate rigid body rotations, an energy map can be obtained as a function of $\boldsymbol{C}$ components $C_{13}$ and $C_{23}$. This landscape is represented in Figure 9a, where the global energy minimum at zero deformation is indicated by the label $\alpha$-TATB. By periodicity, when $\boldsymbol{f}$ equals $\left[\begin{array}{lll}1 & 0 & 0\end{array}\right],\left[\begin{array}{lll}0 & 1 & 0\end{array}\right]$ or a composition of both, the initial structure is restored.

In the vicinity of the initial energy well, only one stable state can be pointed out. The 

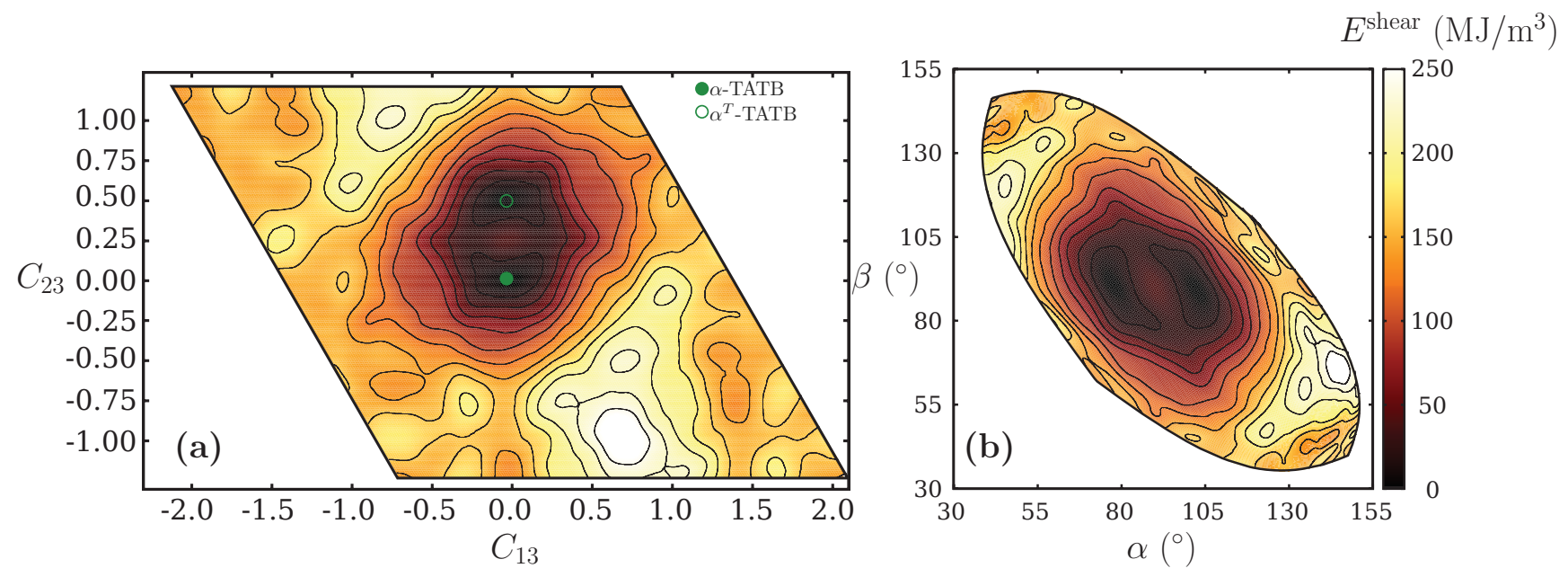

Figure 9: Homogeneous shear energy surfaces for TATB basal plane. (a) Shear energy as a function of the right Cauchy-Green tensor $\boldsymbol{C}$ components: $E^{\text {shear }}=g\left(C_{13}, C_{23}\right)$. (b) Shear energy as a function of out-of-plane angles: $E^{\text {shear }}=g(\alpha, \beta)$.

twinning deformation path, described in the previous section, leads to a stable state, denoted $\alpha^{T}$-TATB. One can see from in Figure $8 \mathrm{~b}$ that this state corresponds to an inversion of the lattice angles $\alpha$ and $\beta$.

An estimate of the stress needed to overcome the energy barrier between the stable an twinning phases can be deduced from the potential $E$ calculated above. By noting that the stress power by unit volume $w$ is $w=\boldsymbol{P}: \dot{\boldsymbol{F}}$ with $\boldsymbol{P}$ the first Piola-Kirchhoff tensor, defined by $\boldsymbol{P}=\partial E / \partial \boldsymbol{F}$, the only two components of the stress active during the transformation are $P_{13}$ and $P_{23}$, by construction of the deformation gradient tensor $\boldsymbol{F}$. We denote this stress $\boldsymbol{\tau}=\left[P_{13}, P_{23}\right]$ and its norm $\tau$. Due to the pure shear nature of the transformation, $P_{13}=\sigma_{13}$ and $P_{23}=\sigma_{23}$ with $\boldsymbol{\sigma}=\boldsymbol{F} \cdot \boldsymbol{P}^{T}$ (the Cauchy stress tensor). Therefore, $\boldsymbol{\tau}$ has the meaning of a resolved stress. The minimum energy paths (MEPs) link strain-free and twinned configurations such as the force derived from the potential is tangent to the MEP. They are calculated with the string method, ${ }^{35}$ a method based on the energy landscape gradient computation. An evaluation of the stress along these MEPs gives a maximum shear stress of $\approx 150 \mathrm{MPa}$. 


\subsubsection{Behavior Under Pure Compression}

When the global deformation contains a prevailing compressive component, we observe an alternating disorientation of the TATB molecular layers from their original position, resulting in a structure buckling in which interfaces are nearly perpendicular to the loading direction. The deformations prescribed in this section are simple-compressive in the basal plane, and therefore different to the isochoric deformation used in Section 3.1 (where it was applied to the study of the onset of plasticity under shear loading). Thus, we apply pure compression to the molecular layers with $\phi=0^{\circ}$ and the deformation gradient tensor is constructed from the vector $\boldsymbol{m}$ only.

We define an orthonormal basis $(\boldsymbol{x}, \boldsymbol{y}, \boldsymbol{z})$ with $\boldsymbol{x}$ parallel to [100] and $\boldsymbol{z}$ parallel to the basal plane normal and the direction of compression by $\boldsymbol{m}=(\cos \theta, \sin \theta, 0)$ with $\theta \in[0: 2 \pi]$. Two system sizes were studied $(40 \boldsymbol{a} \times 40 \boldsymbol{b} \times 10 \boldsymbol{c}$ and $100 \boldsymbol{a} \times 100 \boldsymbol{b} \times 10 \boldsymbol{c})$. No noticeable effects on the observed mechanisms were observed.

Two types of simulations were undertaken: the first consisted in applying an instantaneous compression ratio $R$ followed by an equilibration of the system energy in the NVT ensemble, which allows to observe the arrangement of the molecular layers and its dependence on the compression ratio $R$ and direction $\boldsymbol{m}$. The second type of simulation involved a first step of equilibration in the NVT ensemble for $50 \mathrm{ps}$ followed by a constant strain rate deformation up to a chosen compression ratio $R=5 \%$ which permits to study the strain rate dependence of the system.

\section{Effect of Strain Rate}

We consider the case of prescribed deformation for several constant strain rates, corresponding to a final strain of $5 \%$ with trajectory lengths between 50 ps and 500 ps. In Figure 10 are presented the stress-strain curves for a loading along $\boldsymbol{m}$ with $\theta=30^{\circ}$ and strain rates in $\left[1 \times 10^{8} \mathrm{~s}^{-1}, 1 \times 10^{10} \mathrm{~s}^{-1}\right]$ showing a decrease of the stress to nucleation for decreasing strain rate. No effect of the compression angle $\theta$ was observed for the nucleation stress. 

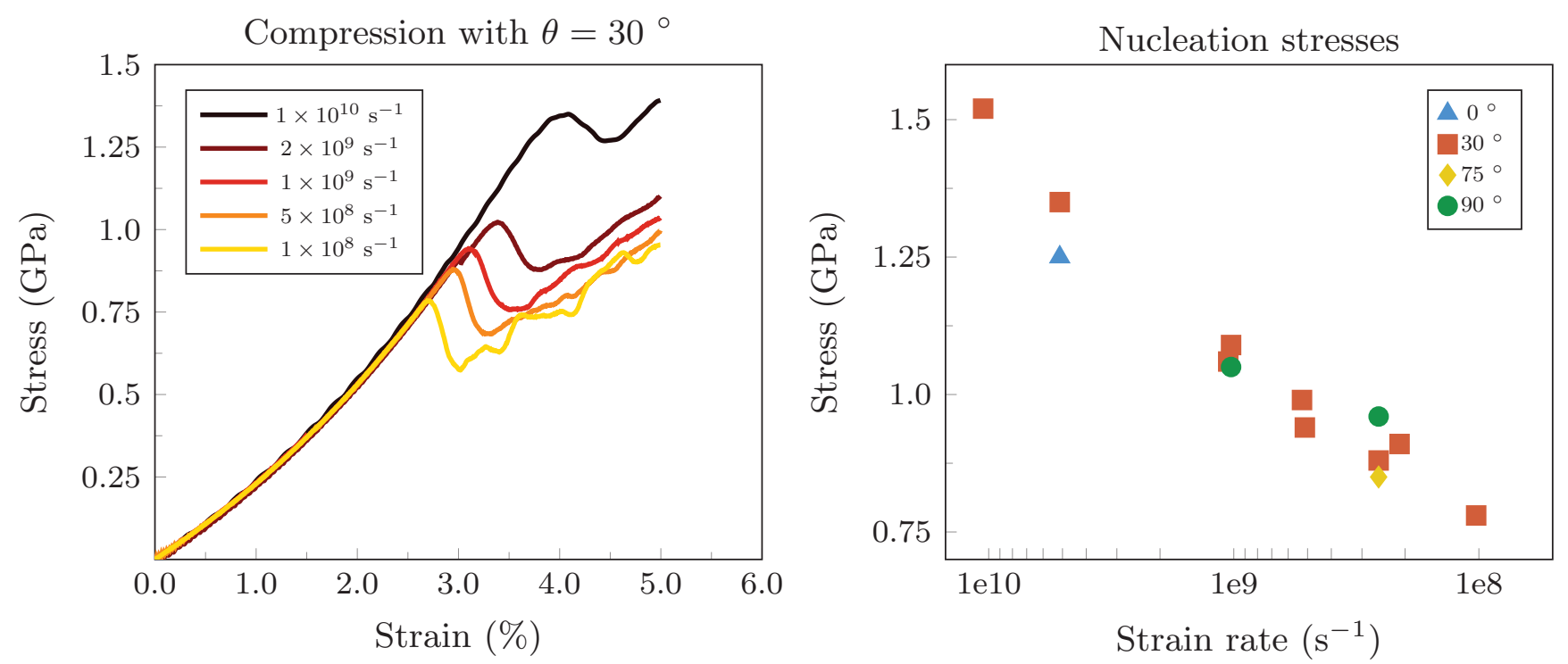

Figure 10: Effect of strain rate on the nucleation stresses. (left) Strain-rate curves for a compression along direction $\boldsymbol{m}$ with $\theta=30^{\circ}$. (right) Different values of the nucleation von Mises stress at nucleation as a function of strain rate, for different directions of compression.

In Figure 11 are shown the map of $\|\boldsymbol{E}\|$ for the final microstructures (system size is $100 \boldsymbol{a} \times 100 \boldsymbol{b} \times 10 \boldsymbol{c})$ for different values of the strain rate, $\|\boldsymbol{E}\|$ being a strain measure with $\boldsymbol{E}=\frac{1}{2}\left(\boldsymbol{F}^{\mathrm{T}} \boldsymbol{F}-\boldsymbol{I}\right)$ the Green-Lagrange strain tensor computed from the local deformation gradient tensor. The increase of strain rate leads to higher nucleation stresses before stress relaxation due to a large number of nucleation sites, compared to what is observed at lower strain rates. This is consistent with a hypothesis of a nucleation rate driven by thermal activation, with the activation energy decreasing with increasing local stress. Each nucleation site produces a chevron that will relax the stress in a zone increasing with time. Such a competition between nucleation and screening is usually well described by the Avrami model ${ }^{36}$ that predicts a decrease of the characteristic length for an increasing nucleation rate, as observed in Figure 11. In the same way, the size of the large deformation bands (denoted in the following as "chevrons") in the microstructure is also related to the strain rate, with larger chevrons at lower strain rates. For the lowest strain rate, we observed a very progressive growth of few chevrons toward a typical converged length of about 50-60 TATB lattices. However, this length is certainly a finite-size effect since the remaining chevrons are disconnected and their merging stops when the entire interface between chevrons becomes 


\section{Norm of the Green-Lagrange Strain Tensor $\|\mathrm{E}\|$}
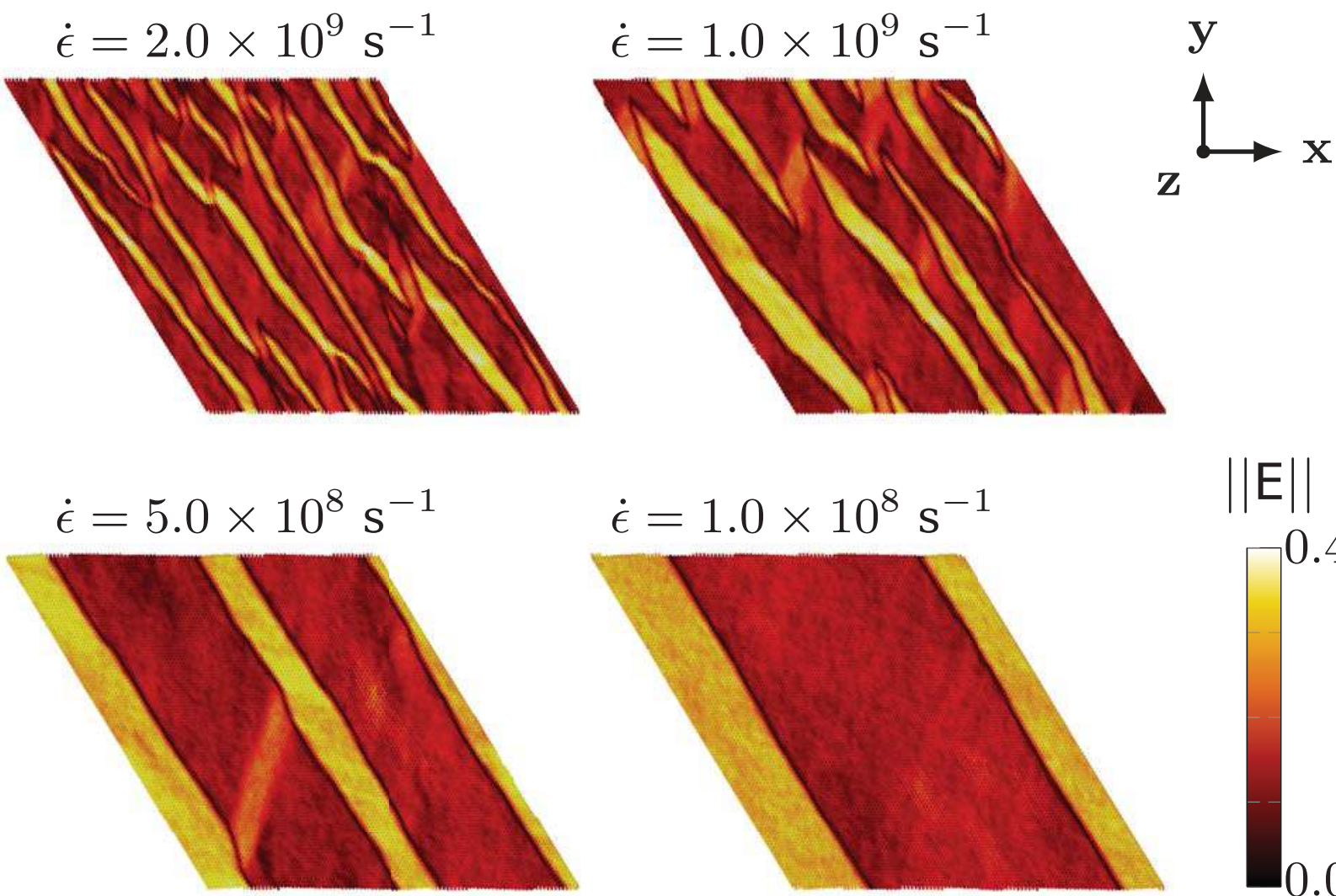

$\dot{\epsilon}=1.0 \times 10^{8} \mathrm{~s}^{-1}$

$\|\mathrm{E}\|$

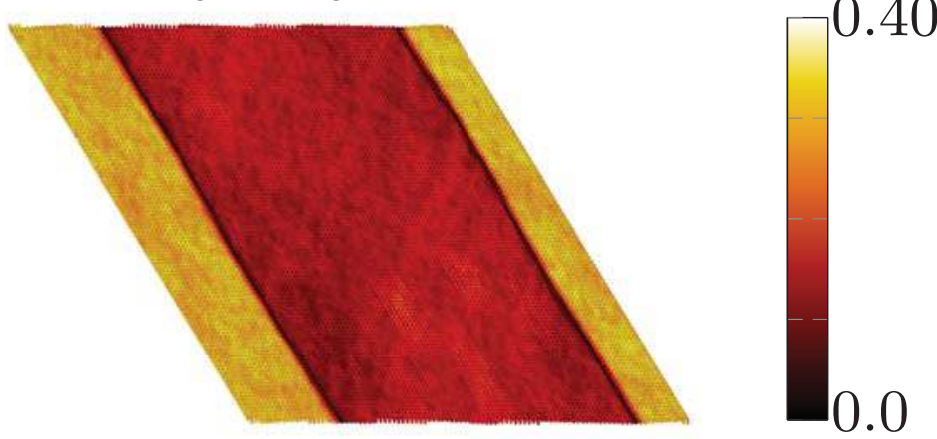

Figure 11: Microstructure evolution with respect to the strain rate. The final microstructure for 4 different molecular dynamics simulations are shown. Each snapshot corresponds to a compression along $\boldsymbol{m}$ with $\theta=30^{\circ}$. Color code corresponds to the value of $\|\boldsymbol{E}\|$. 
perpendicular to the loading direction. Thus, a larger system size would lead to larger chevrons.

\section{Dependence on Loading Direction}

Multiple directions of compression were investigated for this study at the same strain rate of $2 \times 10^{8} \mathrm{~s}^{-1}$. The TATB supercell was compressed along the direction $\boldsymbol{m}=(\cos \theta, \sin \theta, 0)$ for $\theta=30,75$ and $90^{\circ}$.

Chevrons are nucleated with interfaces perpendicular to the compression direction as shown in Figure 12. Snapshots of three molecular dynamics simulations (different rows) are represented, corresponding to different compression angle $\theta\left(30,75\right.$ and $\left.90^{\circ}\right)$, and intermediate strains (3, 4 and 5\%). Starting from an equilibrated and defect-free single crystal, the trigger of the first disorientations happens around $2 \%$ strain, with a homogeneous nucleation of tiny chevrons. As the deformation progresses, a hierarchical evolution is observed with the merging of chevrons to form larger structure. In our case, the TATB sample size was $100 \times 100 \times 10$ lattices. Once the structures were equilibrated, interfaces were constrained by the 3D periodic cell and the growth could not continue.

\section{Microstructure Evolution}

The buckling involves significant shear of the basal plane. In this section, we assess the role of the energy landscape calculated for simple shear in Sections 3.3.1 and 3.3.2 on the formation of the microstructure. As a first step, we estimate the buckling threshold as the highest deformation for which no microstructure appears. This threshold is estimated by instantaneously imposing a deformation, run NVT-MD for 500 ps, and observe a possible onset of microstructure. For a deformation angle of $\theta=0^{\circ}$, the buckling strain threshold is $T_{B}=2.75 \% \pm 0.25$, other angles leading to roughly the same value.

For the results presented in the following, we focus on the evolution of the microstructure during a progressive deformation, at different strain rates. During each simulation, the 
Norm of the Green-Lagrange Strain Tensor $\|\mathrm{E}\|$
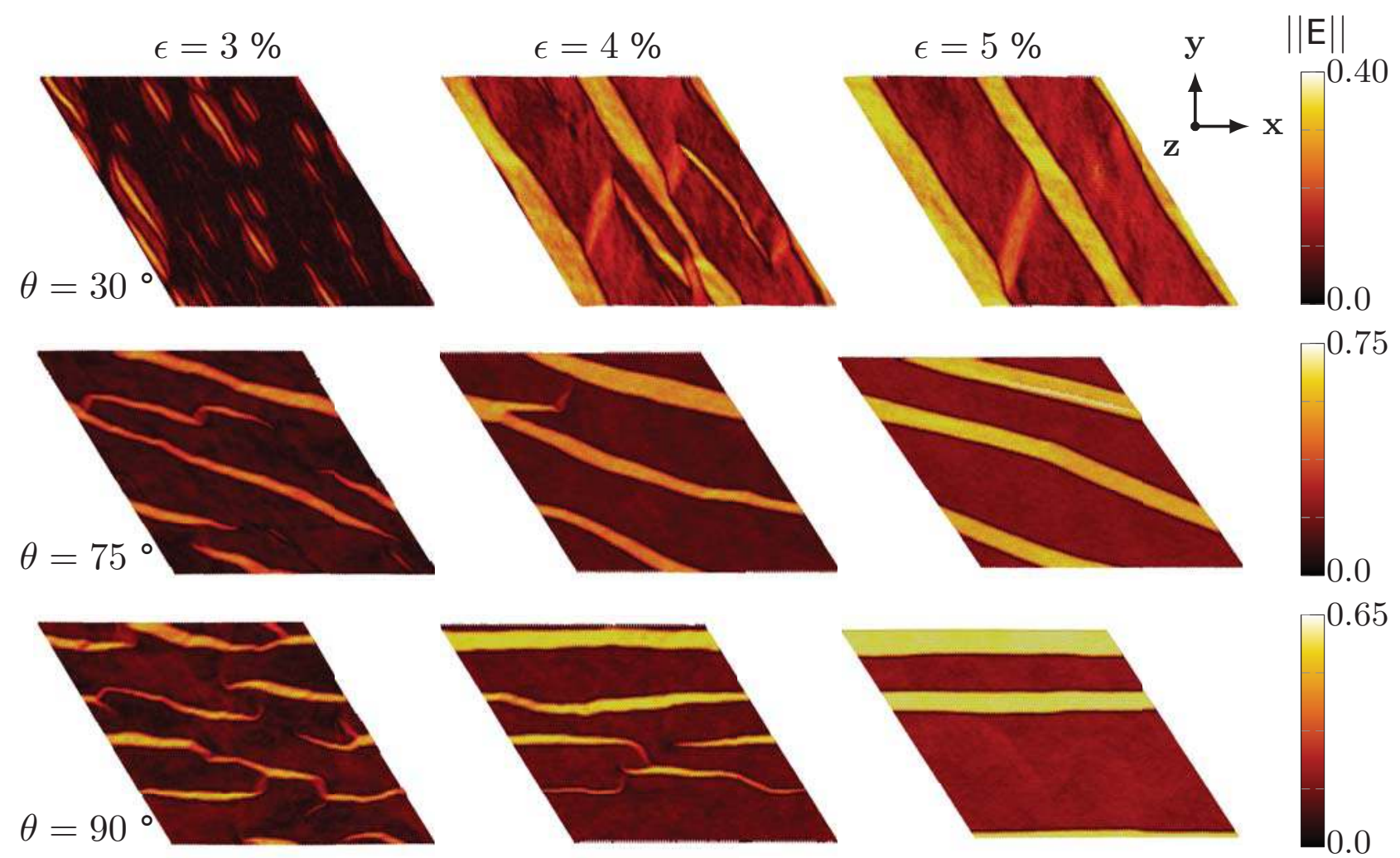

Figure 12: Microstructure evolution with respect to the compression direction. (rows) Snapshots for three different values of the angle $\theta$. (columns) Snapshots for three different values of the strain along the prescribed deformation path simulation. Color code corresponds to the value of $\|\boldsymbol{E}\|$. 
computation of the local deformation gradient tensor $\boldsymbol{F}$ was carried out, as long as the right Cauchy-Green tensor satisfied by $\boldsymbol{C}=\boldsymbol{F}^{\mathrm{T}} \boldsymbol{F}$. For simple shear the only non-trivial components of $\boldsymbol{C}$ are $C_{13}$ and $C_{23}$. The couple $\left(C_{13}, C_{23}\right)$ is computed for every molecule to define a density map $d\left(C_{13}, C_{23}\right)$, so that the number of molecules with deformation of $\left(C_{13} \pm \frac{\Delta}{2}, C_{23} \pm \frac{\Delta}{2}\right)$ is given by $d\left(C_{13}, C_{23}\right) \times \Delta^{2}$.

This density map is then simplified by extracting the maxima and lumping the remaining density to the nearest maximum. This procedure produces a simplified histogram as a list of maxima, each one being associated with a representative fraction of molecules affected by the neighboring strain. By reporting these several pairs of the Cauchy-Green tensor components onto this energy map, we can evaluate whether the deformation signatures correspond to remarkable locations on the energy landscape, e.g., minima. Since the buckling deformation mechanism mainly involves basal plane shear, the analysis of the couple $\left(C_{13}, C_{23}\right)$ is important if one wants to link the buckled microstructure with the twinning energy landscape presented above (Section 3.3.2). For the simulations presented in Figure 13, the plotted sets of the simplified histogram represents more than $92 \%$ of the system.

In Figure 13, we have reported the simplified histogram calculated at the end of the MD simulations, for compressions with $\theta=30,75$ and $90^{\circ}$ and a final strain equal to $5 \%$. The histograms are represented by filled circles whose area is proportional to the fraction of the molecules in this state. For direction with $\theta=30^{\circ}$, the deformation is mostly in $C_{13}$ and the sets are aligned with the direction of loading. In a similar way, when $\theta=90^{\circ}$, the two sets are also aligned with the direction of loading but most of the deformation is in $C_{23}$. Concerning the case when $\theta=75^{\circ}$, the furthest set from the strain-free minimum is split into two subsets with one falling near in the direction of $90^{\circ}$. These observations show that when the direction of compression is aligned with the two minima (i.e. $\theta=90^{\circ}$ ), the microstructure is driven by Twinning, with a strong influence of the underlying energy landscape. Conversely, the more the direction of compression is aligned perpendicularly to the two minima (i.e. $\theta=0^{\circ}$ ), the more the microstructure will be dominated by pure elasticity. We termed this "in-between" 


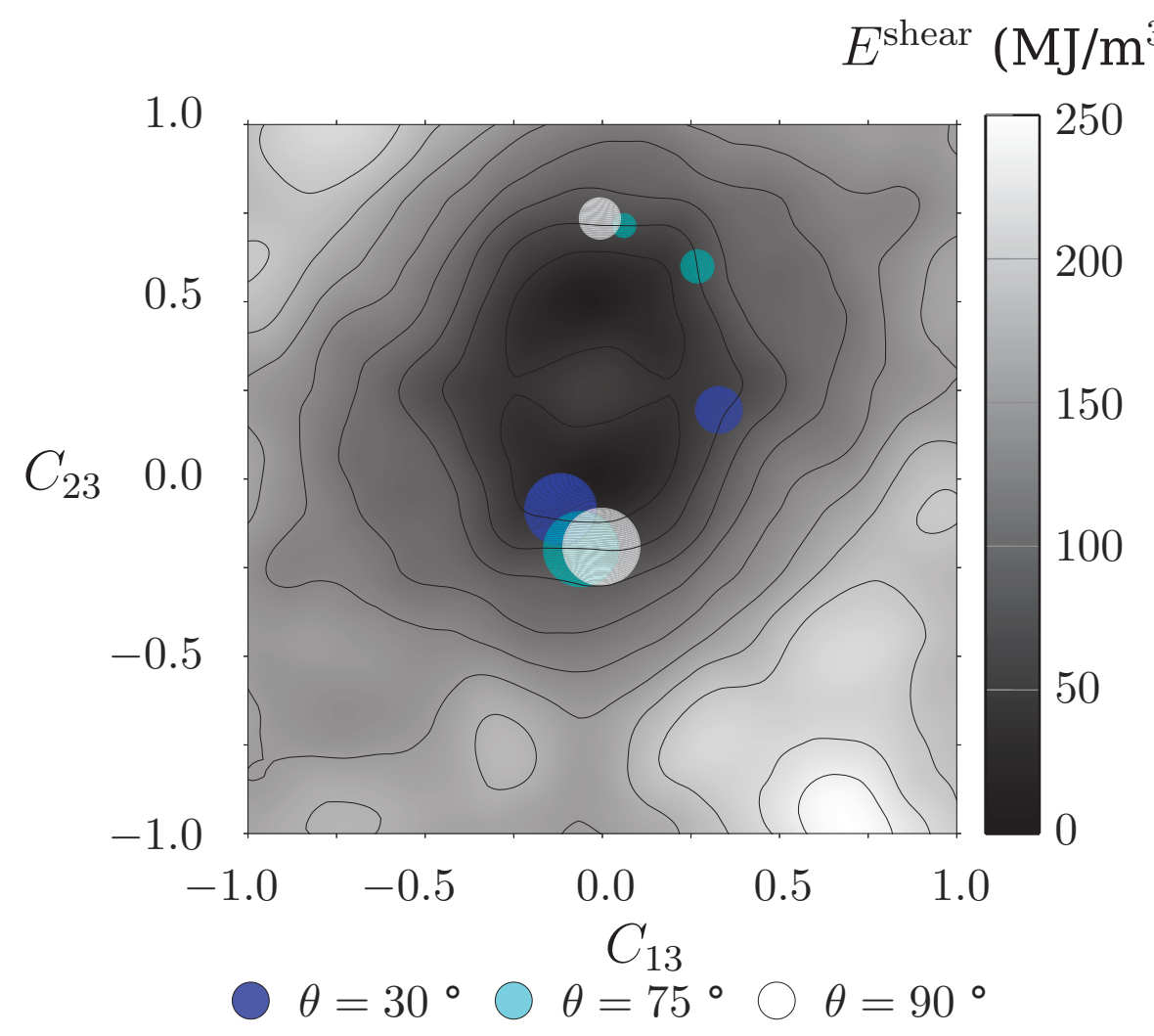

Figure 13: Link between the homogeneous shear energy surface and the deformation signatures obtained during directional deformations. The gray color bar represents the shear energy (similar to Figure 9) while the filled circles represents the deformation signatures, each color corresponding to a different direction of compression. 
mechanism Twinning-Buckling.

The sets fall in the vicinity of the deepest energy well, confirming the role of the underlying energy landscape on the structure. This can be understood by comparing the excess energy for the minimum energy paths (MEPs) between two minima (see Section 3.3.2), estimated at $25.4 \mathrm{MJ} \mathrm{m}^{-3}$, and the elastic energy available prior to the onset of buckling. This latter quantity is the elastic energy to be released by the buckling by considering the elastic energy stored by a deformation of $2.75 \%$ (the buckling threshold $T_{B}$ ) applied under

uniaxial compression: $E_{B}=\frac{1}{2} C_{11} T_{B}^{2}=18.9 \mathrm{MJ} \mathrm{m}^{-3}$. The two energies are therefore similar, demonstrating that even a small compression of a few percent is sufficient to overcome the energy barrier leading to the twinned configuration. Furthermore, if only one minimum had been present on the energy landscape (with a classical quadratic elastic well), two sets with deformations of opposite signs and exactly same amplitude would have been observed, which is not the case here. This implies the necessity to consider the Twinning energy landscape during the construction of a constitutive law that would aim to model the Twinning-buckling mechanism. Relevant inputs include the energy maximum and the energies along the MEP that link the $\alpha$ and $\alpha^{T}$ structures.

\section{CONCLUSIONS}

The computation of the directional nucleation stress surface under pure shear for TATB single crystal through MD simulations allowed us to identify three distinct behaviors of the material. Gliding in the basal plane under pure shear is very easy to activate and is related to the very low energies on the $\gamma$-surface in these directions. This is consistent with a previous study of dislocation core structures for this plane. ${ }^{7}$ Non-basal plane dislocations nucleation is a new plasticity mechanism for the TATB single crystal. It is shown to involve hydrogen-bond breaking and complex dislocation core structures with modification of the hexagonal in-plane molecular packing (for partial dislocations), and a noticeable core 
dilatation. Finally, when the strain consists mainly of compression of the molecular layers, a Twinning-buckling phenomena is inescapably involved, leading to a modification of the microstructure in a succession of chevrons of opposite deformations, with interfaces always normal to the compression direction.

The calculation of a Twinning energy landscape has also been performed. It revealed an energy minimum corresponding to a perfect twinning of the TATB single crystal. The transition between buckling and twinning is dependent on this energy landscape and the direction of loading.

We have shown that TATB single crystal behavior under large strains is very dependent on the loading direction: out-of-plane loading leads to dislocations nucleation, in-plane loadings systematically produce Twinning-buckling whereas basal plane pure shear leads to simple homogeneous gliding. All these elements are the necessary ingredients to build a representative constitutive law for the TATB single crystal behavior under large strains, which ideally could take in account dislocations nucleation, perfect lattice twinning, and buckling mechanism.

\section{ACKNOWLEDGMENTS}

The authors thank Dr. H. Trumel, Dr. O. Castelnau, and Dr. K. Derrien for helpful discussions during the elaboration of this work.

The authors declare no competing financial interest.

\section{APPENDIX: LOCAL COMPUTATION OF THE DEFOR- MATION GRADIENT TENSOR}

In continuum mechanics, the position at time $t$ in the strained state refers to the position in a reference state through the deformation gradient tensor $\boldsymbol{F}(t)$ with $\boldsymbol{x}(t)=\boldsymbol{F}(t) \cdot \boldsymbol{X}$, 
where $\boldsymbol{x}$ and $\boldsymbol{X}$ are the current and initial positions, respectively. We approximate the deformation gradient tensor $\boldsymbol{F}(t)$ by a tensor $\hat{\boldsymbol{F}}_{i}$ defined for each molecule by using the relative displacement of its neighboring molecules.

A pair of particles $i$ and $j$ initially separated by a vector $\boldsymbol{\Delta} \boldsymbol{X}_{i j}$ from each other, are currently distant by $\boldsymbol{\Delta} \boldsymbol{x}_{i j}$ with $\boldsymbol{\Delta} \boldsymbol{x}_{i j} \approx \hat{\boldsymbol{F}}_{i} \cdot \boldsymbol{\Delta} \boldsymbol{X}_{i j}$. The corresponding error function noted $\chi_{i j}^{2}$ reads:

$$
\chi_{i j}^{2}=\left(\boldsymbol{\Delta} \boldsymbol{x}_{i j}-\hat{\boldsymbol{F}}_{i} \cdot \boldsymbol{\Delta} \boldsymbol{X}_{i j}\right)^{\mathrm{T}}\left(\boldsymbol{\Delta} \boldsymbol{x}_{i j}-\hat{\boldsymbol{F}}_{i} \cdot \boldsymbol{\Delta} \boldsymbol{X}_{i j}\right)
$$

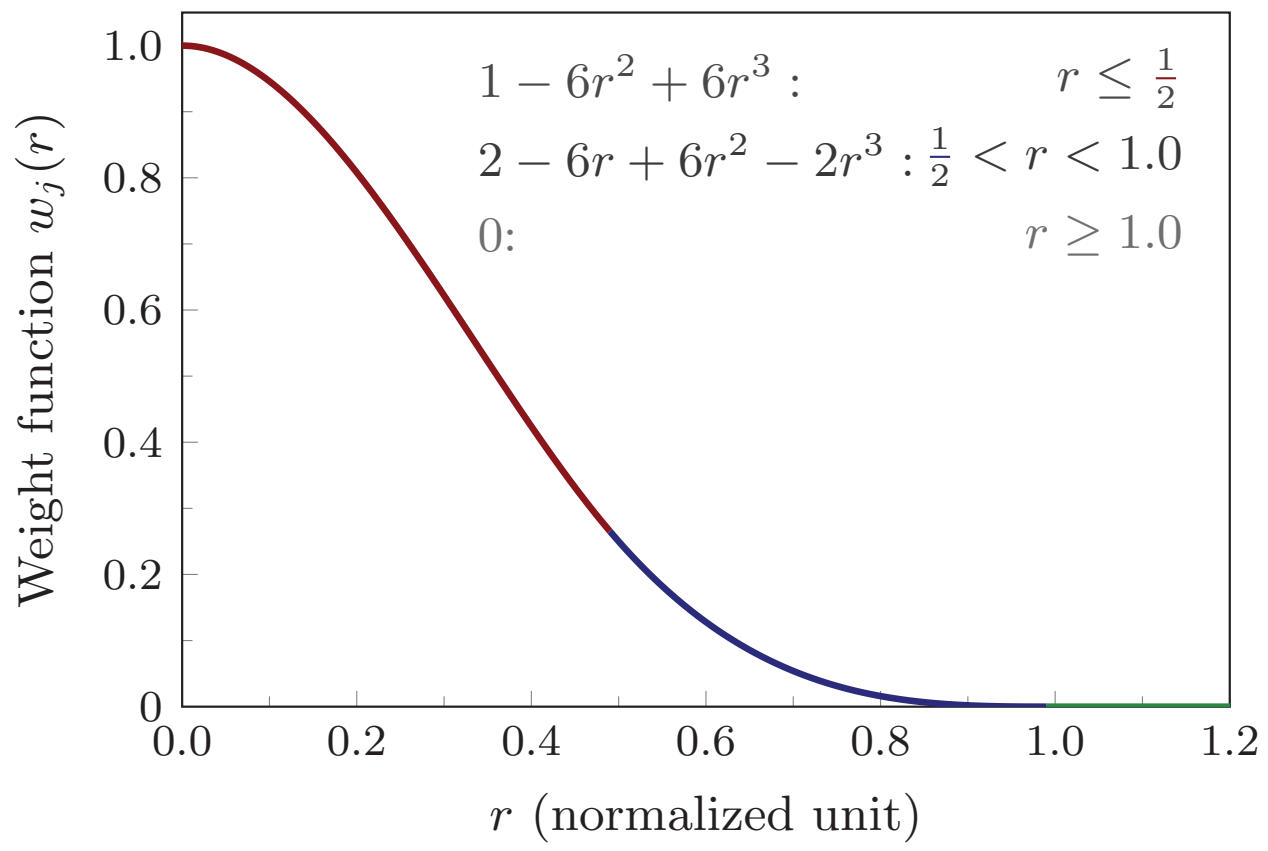

Figure 14: Weight function $w_{j}(r)$ used for the computation of the local deformation gradient tensor with $r$ normalized by the cutoff radius $r_{\text {cut }}$ used for the measure. In this work, $r_{\text {cut }}=26 \AA$.

Thus, for a number $N$ of neighbors in a sphere of radius $r_{\text {cut }}$ around the particle $i$, the total weighted least squares error is:

$$
\chi_{i}^{2}=\sum_{j=1}^{N} \chi_{i j}^{2} w_{j}
$$

where $w_{j}$ is a weight coefficient associated to the $j^{\text {th }}$ neighbor varying from 1 to 0 for $\left\|\boldsymbol{\Delta} \boldsymbol{x}_{i j}\right\|=0$ to $r_{\text {cut }}$ using a cubic spline function. This function is represented in Figure 14 
with abscissa $r=\left\|\boldsymbol{\Delta} \boldsymbol{x}_{i j}\right\| / r_{\text {cut }}$. Here, $r_{\text {cut }}$ is the cutoff radius used for the local measure of the deformation and equals two times the cutoff radius of the potential so that a sufficient number of neighbors are taken into account to perform the measure. The minimization of $\chi_{i}$ with respect to the $\hat{\boldsymbol{F}}$ components leads to the following relation:

$$
\hat{\boldsymbol{F}}_{i}=\sum_{j=1}^{N} \boldsymbol{\Delta} \boldsymbol{x}_{i j} \boldsymbol{\Delta} \boldsymbol{X}_{i j}^{\mathrm{T}} w_{j}\left(\sum_{j=1}^{N} \boldsymbol{\Delta} \boldsymbol{X}_{i j} \boldsymbol{\Delta} \boldsymbol{X}_{i j}^{\mathrm{T}} w_{j}\right)^{-1}=\boldsymbol{A}_{i} \boldsymbol{D}_{i}^{-1}
$$

where $\boldsymbol{A}_{i}$ and $\boldsymbol{D}_{i}$ are second order tensors. $\boldsymbol{D}_{i}$ refers to the initial state, and $\boldsymbol{A}_{i}$ links both current and reference states. From there, through a simple polar decomposition, one can compute the local rotation tensor $\hat{\boldsymbol{R}}_{i}$ and the local stretch tensor $\hat{\boldsymbol{U}}_{i}$. This decomposition allows to identify rigid body rotations during MD simulations through $\hat{\boldsymbol{R}}_{i}$ and also the computation of the local lattice parameters and angles by tensorial operation on $\hat{\boldsymbol{U}}_{i}$. Since we used rigid molecules in this work, $i$ and $j$ in the precedent equations stand for the molecular centers of mass. These objects are used and analyzed in Section 3.

\section{References}

(1) Cady, H.H.; Larson, A.C., The Crystal Structure of 1,3,5-triamino-2,4,6trinitrobenzene. Acta. Crys. 1965, 18, 485-496.

(2) Plisson, T.; Pineau, N.; Weck, G.; Bruneton, E.; Guignot, N.; Loubeyre, P., Equation of State of 1,3,5-triamino-2,4,6-trinitrobenzene up to 66 GPa. J. Appl. Phys. 2017, 122, 235901.

(3) Kroonblawd, M.P.; Sewell, T.D., Theoretical Determination of Anisotropic Thermal Conductivity for Crystalline 1,3,5-triamino-2,4,6-trinitrobenzene (TATB). J. Chem. Phys. 2013, 139.

(4) Kroonblawd, M.P.; Sewell, T.D., Theoretical Determination of Anisotropic Thermal Conductivity for Initially Defect-Free and Defective TATB Single Crystals. J. Chem. 
Phys. 2014, 141.

(5) Bedrov, D.; Borodin O.; Smith, G.D.; Sewell, T.D.; Dattelbaum, D.M.; Stevens, L.L., A Molecular Dynamics Simulation Study of Crystalline 1,3,5-triamino-2,4,6trinitrobenzene as a Function of Pressure and Temperature. J. Chem. Phys. 2009, 131.

(6) Mathew, N.; Sewell, T.D., Generalized Stacking Fault Energies in the Basal Plane of Triclinic Molecular Crystal 1,3,5-triamino-2,4,6-trinitrobenzene. Philos. Mag. 2015, 95, $424-440$.

(7) Lafourcade, P.; Denoual, C.; Maillet, J.-B., Dislocation Core Structure at Finite Temperature Inferred by Molecular Dynamics Simulations for 1,3,5-Triamino-2,4,6Trinitrobenzene Single Crystal. J. Phys. Chem. C 2017, 121, 7442-7449.

(8) Phillips, D.S.; Schwarz, R.B.; Skidmore, C.B.; Hiskey, M.A.; Son, S.F., Some Observations on the Structure of TATB. American Institute of Physics Conference Series. 2000; pp 707-710.

(9) Hoffman, D.M.; Trevor M.W.; Mitchell, A.R.; Depiero, S.C., Comparison of New and Legacy TATBs. J. Energ. Mat. 2008, 26, 139-162.

(10) Hoffman, D.M; Fontes, A.T., Density Distributions in TATB Prepared by Various Methods. Propellants, Explos., Pyrotech. 2010, 35, 15-23.

(11) Mallik, B.S.; Kuo, I-F.W.; Fried, L.E.; Siepmann, J.I., Understanding the Solubility of Triamino-Trinitrobenzene in Hydrous Tetramethylammonium Fluoride: A First Principles Molecular Dynamics Simulation Study. Phys. Chem. Chem. Phys. 2012, 14, 4884-4890.

(12) Luscher, D.J.; Yeager, J.D.; Clausen, B.; Vogel, S.C.; Higginbotham D.; Amanda L.; Brown, D.W., Using Neutron Diffraction to Investigate Texture Evolution During Con- 
solidation of Deuterated Triaminotrinitrobenzene (d-TATB) Explosive Powder. Crystals 2017,7 .

(13) Taw, M.R.; Yeager, J.D.; Hooks, D.E.; Carvajal, T.M.; Bahr, D.F., The Mechanical Properties of As-grown Noncubic Organic Molecular Crystals Assessed by Nanoindentation. J. Mater. Res. 2017, 1-10.

(14) Kroonblawd, M.P.; Sewell, T.D.; Maillet, J-B., Characteristics of Energy Exchange Between Inter- and Intramolecular Degrees of Freedom in Crystalline 1,3,5-triamino-2,4,6trinitrobenzene (TATB) with Implications for Coarse-grained Simulations of Shock Waves in Polyatomic Molecular Crystals. J. Chem. Phys. 2016, 144.

(15) Mathew, N.; Sewell, T.D., Nanoindentation of the Triclinic Molecular Crystal 1,3,5Triamino-2,4,6-trinitrobenzene: A Molecular Dynamics Study. J. Phys. Chem. C 2016, 120, 8266-8277.

(16) Davis, W. C., High Explosives: The Interaction of Chemistry and Mechanics. Los Alamos Sci. 1981,

(17) Field, J.E., Hot Spot Ignition Mechanisms for Explosives. Acc. Chem. Res. 1992, 25, 489-496.

(18) Armstrong, R.W.; Ammon, H.L.; Elban, W.L.; Tsai, D.H., Investigation of Hot Spot Characteristics in Energetic Crystals. Thermochim. Acta 2002, 384, $303-313$.

(19) An, Q; Goddard, W.A.; Zybin, S.V.;Jaramillo-Botero, A.; Zhou, T., Highly Shocked Polymer Bonded Explosives at a Nonplanar Interface: Hot-Spot Formation Leading to Detonation. J. Phys. Chem. C 2013, 117, 26551-26561.

(20) Eason, R.M.; Sewell, T.D., Molecular Dynamics Simulations of the Collapse of a Cylindrical Pore in the Energetic Material $\alpha$-RDX. J. Dyn. Behav. Mat. 2015, 1, 423-438. 
(21) Austin, R.A.; Barton, N.R.; Reaugh, J.E.; Fried, L.E., Direct Numerical Simulation of Shear Localization and Decomposition Reactions in Shock-loaded HMX Crystal. J. Appl. Phys. 2015, 117.

(22) Soulard, L., Molecular Dynamics Study of the Micro-spallation. Eur. Phys. J. D 2008, 50.

(23) Onsager, L., Electric Moments of Molecules in Liquids. J. Am. Chem. Soc. 1936, 58, 1486-1493.

(24) Barker, J.A.; Watts, R.O., Monte Carlo Studies of the Dielectric Properties of Waterlike Models. Mol. Phys. 1973, 26, 789-792.

(25) Watts, R.O., Monte Carlo Studies of Liquid Water. Mol. Phys. 1974, 28, 1069-1083.

(26) Verlet, L., Computer "Experiments" on Classical Fluids. II. Equilibrium Correlation Functions. Phys. Rev. 1968, 165, 201-214.

(27) Kroonblawd, M. P.; Sewell, T. D. Anisotropic Relaxation of Idealized Hot Spots in Crystalline 1,3,5-Triamino-2,4,6-trinitrobenzene (TATB). J. Phys. Chem. C 2016, 120, 17214-17223.

(28) Lafourcade, P.; Denoual, C.; Maillet, J.-B., Detection of Plasticity Mechanisms in an Energetic Molecular Crystal Through Shock-like 3D Unidirectional Compressions: A Molecular Dynamics Study. APS Shock Compression of Condensed Matter Meeting Abstracts. 2017; p H8.002.

(29) Stukowski, A.; Albe, L., Extracting Dislocations and Non-dislocation Crystal Defects from Atomistic Simulation Data. Modell. Simul. Mater. Sci. Eng. 2010, 18, 085001.

(30) Gullett, P.M.; Horstemeyer, M.F.; Baskes, M.I.; Fang, H., A Deformation Gradient Tensor and Strain Tensors for Atomistic Simulations. Modell. Simul. Mater. Sci. Eng. 2008,16 . 
(31) Tucker, G.J.; Zimmerman, J.A.; McDowell, D.L., Continuum Metrics for Deformation and Microrotation from Atomistic Simulations: Application to Grain Boundaries. Int. J. Eng. Sci. 2011, 49, $1424-1434$.

(32) Denoual, C.; Caucci, A.-M.; Soulard, L.; Pellegrini, Y.-P., Phase-Field ReactionPathway Kinetics of Martensitic Transformations in a Model Fe ${ }_{3} \mathrm{Ni}$ Alloy. Phys. Rev. Lett. 2010, 105, 035703.

(33) Kober, E.; Mathew, N.; Rudin, S., Characterizing Atomistic Geometries and Potential Functions Using Strain Functionals. APS Shock Compression of Condensed Matter Meeting Abstracts. 2017; p B5.001.

(34) Makke, A.; Lame, O.; Perez, M.; Barrat, J.-L., Nanoscale Buckling in Lamellar Block Copolymers: A Molecular Dynamics Simulation Approach. Macromolecules 2013, 46, $7853-7864$.

(35) Weinan, E.; Weiqing, R.; Vanden-Eijnden E., Simplified and Improved String Method for Computing the Minimum Energy Paths in Barrier-crossing Events. J. Chem. Phys. 2007, 126, 164103.

(36) Avrami, M., Kinetics of Phase Change. I General Theory. J. Chem. Phys. 1939, 7, $1103-1112$. 